Publ. RIMS Kyoto Univ. 46 (2010), 1,35

DOI 10.2977/PRIMS/1

\title{
A Simpson Correspondence in Positive Characteristic
}

by

\author{
Michel Gros, Bernard Le Stum and Adolfo Quirós
}

\begin{abstract}
Let $p$ be a prime number, $k$ a perfect field of characteristic $p>0, X$ a smooth $k$-scheme and $\mathcal{D}_{X}^{(m)}$ the algebra of (arithmetic) differential operators of level $m \geq 0$. We study the Azumaya nature of this algebra and show how to construct, using an additional data, a splitting of a completion (along some ideal contained in its center) of it.
\end{abstract}

2010 Mathematics Subject Classification: Primary 14F30; Secondary 14F40.

Keywords: $\mathcal{D}$-module, Higgs field, connection, $p$-curvature, Frobenius.

\section{Introduction}

The first two authors had the opportunity to participate in a working group in Rennes dedicated to the work of Arthur Ogus and Vadim Vologodsky on nonabelian Hodge theory, which is now published in [11. This is an analog in positive characteristic $p$ of Simpson's correspondence over the complex numbers between local systems and a certain type of holomorphic vector bundles that he called Higgs bundles ([12]). Actually, Pierre Berthelot had previous results related to these questions and he used this opportunity to explain them to us. What we want to do here is to extend these results to differential operators of higher level. In the future, we wish to lift the theory modulo some power of $p$ and compare to Faltings-Simpson $p$-adic correspondence ([5]).

We would also like to mention some other papers related to our investigation. First, there is an article [7] by Masaharu Kaneda where he proves the (semi-linear)

Communicated by S. Mochizuki. Received November 13, 2008. Revised April 29, 2009.

M. Gros: IRMAR, Université de Rennes 1, Campus de Beaulieu, 35042 Rennes Cedex, France. e-mail: michel.gros@univ-rennes1.fr

B. Le Stum: IRMAR, Université de Rennes 1, Campus de Beaulieu, 35042 Rennes Cedex, France. e-mail: bernard.le-stum@univ-rennes1.fr

A. Quirós: Departamento de Matemáticas, Universidad Autónoma de Madrid, Ciudad Universitaria de Cantoblanco, E-28049 Madrid, España.

e-mail: adolfo.quiros@uam.es

(c) 2010 Research Institute for Mathematical Sciences, Kyoto University. All rights reserved. 
Azumaya nature of the ring of differential operators of higher level, generalizing the result of [4]. Also, Marius van der Put in [13] studies the (linear) Azumaya nature of differential operators in the context of differential fields.

We will first recall, in Sections 1 and 2, in an informal way the notion of divided powers of higher level and how this leads, using some duality, to arithmetic differential operators. Then, in Section 3 we will define the notion of $p^{m}$-curvature and show that Kaneda's isomorphism still holds over an arbitrary basis. Next, in Section 4, we assume that there exists a strong lifting of Frobenius mod $p^{2}$ and use it to lift the divided Frobenius and derive a Frobenius map on the ring of differential operators of level $m$. Actually, we can do better and prove in Theorem 4.13 that this data determines a splitting of a central completion of this ring. It is then rather formal, in Section 5 , to obtain a Simpson correspondence and we can even give some explicit formulas. We finish the article, in Section 6, with a series of complements concerning compatibility with other theories.

\section{Conventions}

We let $p$ be a prime and $m \in \mathbb{N}$. Actually, we are interested in the $m$-th power of $p$. When $m=0$, we have $p^{m}=1$ which is therefore independent of $p$. We may also consider the case $m=\infty$ in which case we will write $p^{m}=0$. Again, this is independent of $p$.

Unless $m=0$ or $m=\infty$, all schemes are assumed to be $\mathbb{Z}_{(p)}$-schemes.

We use standard multiindex notations, hoping that everything will be clear from the context.

\section{$\S 1$. Usual divided powers}

It seems useful to briefly recall here some basic results on usual divided powers that we will need afterwards. There is nothing new but we hope that it makes the next sections easier to read without referring to older articles. The main point of this section is to clarify the duality between divided powers and regular powers.

Let $\mathcal{R}$ be a commutative ring (in a topos).

Definition 1.1. A divided power structure on an ideal $\mathcal{I}$ in a commutative $\mathcal{R}$ algebra $\mathcal{A}$ is a family of maps

$$
\mathcal{I} \rightarrow A, \quad f \mapsto f^{[k]},
$$

that behave like $f \mapsto f^{k} / k$ !. We then say that $\mathcal{I}$ is a divided power ideal or that $\mathcal{A}$ is a divided power $\mathcal{R}$-algebra. 
We will not list all the required properties. Note however that we always have

$$
f^{[k]} f^{[l]}=\left(\begin{array}{c}
k+l \\
k
\end{array}\right) f^{[k+l]} .
$$

Theorem 1.2. The functor $\mathcal{A} \mapsto \mathcal{I}$ from divided power $\mathcal{R}$-algebras to $\mathcal{R}$-modules has a left adjoint $\mathcal{M} \mapsto \Gamma \cdot \mathcal{M}$.

Proof. See, for example, Theorem 3.9 of [3].

Actually $\Gamma_{\bullet} \mathcal{M}$ is a graded algebra with divided power ideal $\Gamma_{>0} \mathcal{M}$. Not also that $\Gamma \cdot \mathcal{M}$ is generated as $\mathcal{R}$-algebra by all the $s^{[k]}$ for $s \in M$. For example, if $\mathcal{M}$ is free on $\left\{s_{\lambda}: \lambda \in \Lambda\right\}$, then $\Gamma_{k} \mathcal{M}$ is free on the $\underline{s}^{[\underline{k}]}:=\prod s_{\lambda}^{\left[k_{\lambda}\right]}$ with $|\underline{k}|:=\sum k_{\lambda}=k$. Moreover, multiplication is given by the general formula for divided powers recalled above.

Definition 1.3. If $\mathcal{M}$ is an $\mathcal{R}$-module, the ring $\Gamma_{\bullet} \mathcal{M}$ is called the divided power algebra on $\mathcal{M}$.

If $\mathcal{M}$ is an $\mathcal{R}$-module, we will denote by $S^{\bullet} \mathcal{M}$ the symmetric algebra on $\mathcal{M}$ and by $\check{\mathcal{M}}$ the dual of $\mathcal{M}$. Also, we will denote by $\widehat{S^{\bullet \mathcal{M}}}$ the completion of $S^{\bullet} \mathcal{M}$ along $S^{>0} \mathcal{M}$.

Proposition 1.4. If $\mathcal{M}$ is an $\mathcal{R}$-module, there exists a canonical pairing

$$
S^{\bullet} \check{\mathcal{M}} \times \Gamma_{\bullet} \mathcal{M} \rightarrow \mathcal{R}, \quad\left(\varphi_{1} \cdots \varphi_{n}, s^{[n]}\right) \mapsto \varphi_{1}(s) \cdots \varphi_{n}(s),
$$

giving rise to perfect duality at each step when $\mathcal{M}$ is locally free of finite type.

Proof. See, for example, Proposition A.10 of [3].

The general formula for this pairing is quite involved but if $\left\{s_{\lambda}: \lambda \in \Lambda\right\}$ is a finite basis for $\mathcal{M}$, and $\left\{\check{s}_{\lambda}: \lambda \in \Lambda\right\}$ denotes the dual basis, then the dual basis to $\left\{\underline{s}^{\underline{k}}\right\}$ is nothing but $\left\{\underline{s}^{[k]}\right\}$.

Corollary 1.5. If $\mathcal{M}$ is a locally free $\mathcal{R}$-module of finite type, we have a perfect pairing

$$
\widehat{S_{\bullet} \check{\mathcal{M}}} \times \Gamma \cdot \mathcal{M} \rightarrow \mathcal{R}
$$

Of course, there exists also a natural map $S^{\bullet} \mathcal{M} \rightarrow \Gamma \cdot \mathcal{M}$ but it is not injective in general: if $p^{N+1}=0$ on $X$, then $f^{p^{n}} \mapsto p^{n} f^{\left[p^{n}\right]}=0$ for $n>N$.

Proposition 1.6. If $\mathcal{M}$ is an $\mathcal{R}$-module, multiplication on $S^{\bullet} \check{\mathcal{M}}$ is dual to the diagonal map

$$
\Gamma_{\bullet} \mathcal{M} \stackrel{\delta}{\rightarrow} \Gamma \cdot(\mathcal{M} \oplus \mathcal{M}) \stackrel{\simeq}{\rightarrow} \Gamma_{\bullet} \mathcal{M} \otimes \Gamma \cdot \mathcal{M}, \quad s^{[k]} \mapsto \sum_{i+j=k} s^{[i]} \otimes s^{[j]} .
$$


Proof. The point is to show that $\left(\varphi_{1} \cdots \varphi_{i} \otimes \psi_{1} \cdots \psi_{j}\right) \circ \delta$ acts like $\varphi_{1} \cdots \varphi_{i} \psi_{1} \cdots \psi_{j}$ on $s^{[k]}$ when $k=i+j$. And this is clear.

\section{$\S 2$. Higher divided powers}

We quickly recall the definition of Berthelot's divided powers of level $m$ and how one derives the notion of differential operators of higher level from them (see [1] for a detailed exposition). We stick to a geometric situation.

Definition 2.1. Let $X \hookrightarrow Y$ be an immersion of schemes defined by an ideal $\mathcal{I}$. A divided power structure of level $m$ on $\mathcal{I}$ is a divided power ideal $\mathcal{J} \subset \mathcal{O}_{Y}$ such that

$$
\mathcal{I}^{\left(p^{m}\right)}+p \mathcal{I} \subset \mathcal{J} \subset \mathcal{I}
$$

Here, $\mathcal{I}^{\left(p^{m}\right)}$ denotes the ideal generated by the $p^{m}$-th powers of elements of $\mathcal{I}$. It is then possible to define partial divided powers on $\mathcal{I}$ : they are maps

$$
\mathcal{I} \rightarrow \mathcal{A}, \quad f \mapsto f^{\{k\}},
$$

that behave like $f \mapsto f^{k} / q$ ! where $q$ is the integral part of $k / p^{m}$. Actually, if $k=q p^{m}+r$ and $f \in \mathcal{I}_{X}$, one sets

$$
f^{\{k\}}:=f^{r}\left(f^{p^{m}}\right)^{[q]} .
$$

We have, as above, a multiplication formula (writing $q_{k}$ instead of $q$ in order to take into account the dependence on $k$ ):

$$
f^{\{k\}} f^{\{l\}}=\left\{\begin{array}{c}
k+l \\
k
\end{array}\right\} f^{\{k+l\}} \text { where } \quad\left\{\begin{array}{c}
k+l \\
k
\end{array}\right\}=\frac{q_{k+l} !}{q_{k} ! q_{l} !} .
$$

When $m=0$, we must have $\mathcal{J}=\mathcal{I}$ and $f^{\{k\}}=f^{[k]}$ is the above divided power. When $m=\infty$, the condition reduces to $p \mathcal{I} \subset \mathcal{J} \subset \mathcal{I}$ and we may always choose $\mathcal{J}=\mathcal{I}$ since $p \mathcal{I}$ has divided powers. Also, in this case, $f^{\{k\}}=f^{k}$ is just the usual power.

We fix a (formal) scheme $S$ with a divided power structure of level $m$ on some ideal of $\mathcal{O}_{S}$ and we assume that all constructions below are made over $S$ and are "compatible" with the divided powers on $S$ in a sense that we do not want to make precise here (see [1] for details). Actually, in the case of a regular immersion, the divided power envelope defined below does not depend on $S$ and this applies in particular to the diagonal embedding of a smooth $S$-scheme.

Proposition 2.2. If an immersion $X \hookrightarrow Y$ has divided powers of level $m$, there is a finest (decreasing) ring filtration $\mathcal{I}^{\{n\}}$ such that $\mathcal{I}^{\{1\}}=\mathcal{I}$ and such that $f^{\{h\}} \in \mathcal{I}^{\{n h\}}$ whenever $f \in \mathcal{I}^{\{n\}}$. 
Proof. See Proposition 1.3.7 of [1].

Proposition 2.3. The functor that forgets the divided power structure on an immersion $X \hookrightarrow Y$ has a left adjoint $X \hookrightarrow P_{X, m}(Y)$.

Proof. See Proposition 1.4.1 of [1].

Definition 2.4. If $X \hookrightarrow Y$ is an immersion of schemes, then $P_{X, m}(Y)$ is called the divided power envelope of level $m$ of $X$ in $Y$.

We will denote by $\mathcal{P}_{X, m}(Y)$ the structural sheaf of $P_{X, m}(Y)$ and by $\mathcal{I}_{X, m}(Y)$ the divided power ideal of level $m$. We will also need to consider the usual divided power ideal $\mathcal{J}_{X, m}(Y)$ (in [1, these ideals are denoted by $\overline{\mathcal{I}}$ and $\tilde{\mathcal{I}}$ if $\mathcal{I}$ denotes the ideal that defines the immersion). For each $n$, we will denote by $P_{X, m}^{n}(Y)$ the subscheme defined by $\mathcal{I}_{X, m}^{\{n+1\}}(Y)$ and consider its structural sheaf

$$
\mathcal{P}_{X, m}^{n}(Y)=\mathcal{P}_{X, m}(Y) / \mathcal{I}_{X, m}^{\{n+1\}}(Y) .
$$

We will mainly be concerned with diagonal immersions $X \hookrightarrow X \times_{S} X$, and we will then write $P_{X, m}, \mathcal{P}_{X, m}, \mathcal{I}_{X, m}, \mathcal{J}_{X, m}, P_{X, m}^{n}$ and $\mathcal{P}_{X, m}^{n}$ respectively.

If we are given local coordinates $t_{1}, \ldots, t_{r}$ on $X / S$, the ideal $\mathcal{I}$ of the diagonal immersion is generated by the $\tau_{i}=1 \otimes t_{i}-t_{i} \otimes 1$. We always implicitly use the first projection as structural map and therefore write $t_{i} \otimes 1=t_{i}$ and $1 \otimes t_{i}=t_{i}+\tau_{i}$. When $m=0, \mathcal{P}_{X, m}$ is nothing but the divided power algebra on the free $\mathcal{O}_{X^{-}}$ module on the generators $\tau_{1}, \ldots, \tau_{r}$. Of course, for $m=\infty$, this is just the symmetric algebra.

In general, we obtain

$$
\mathcal{O}_{X}\left\langle\tau_{1}, \ldots, \tau_{r}\right\rangle^{(m)}:=\left\{\sum_{\text {finite }} f_{\underline{i}} \tau^{\{\underline{i}\}}: f_{\underline{i}} \in \mathcal{O}_{X}\right\}
$$

with multiplication given by the general formula for divided powers of level $m$ recalled above.

Definition 2.5. If $X$ is an $S$-scheme, the dual to $\mathcal{P}_{X, m}^{n}$ is the sheaf $\mathcal{D}_{X, n}^{(m)}$ of differential operators of level $m$ and order at most $n$ and $\mathcal{D}_{X}^{(m)}=\bigcup_{n} \mathcal{D}_{X, n}^{(m)}$ is the sheaf of differential operators of level $m$ on $X / S$.

There is a composition law on $\mathcal{D}_{X}^{(m)}$ that comes by duality from the morphism

$$
\mathcal{P}_{X, m} \stackrel{\delta}{\rightarrow} \mathcal{P}_{X, m} \otimes \mathcal{P}_{X, m}, \quad a \otimes b \mapsto(a \otimes 1) \otimes(1 \otimes b) .
$$

When $X / S$ is smooth, this turns $\mathcal{D}_{X}^{(m)}$ into a non-commutative ring. 
Locally, we see that

$$
\mathcal{D}_{X}^{(m)}=\left\{\sum_{\text {finite }} f_{\underline{i}} \partial^{\langle\underline{i}\rangle}: f_{\underline{i}} \in \mathcal{O}_{X}\right\}
$$

where $\underline{\partial}^{\langle\underline{i}\rangle}$ is the dual basis to $\underline{\tau}^{\{\underline{i}\}}$ and multiplication on differentials is given by

$$
\underline{\partial}^{\langle\underline{k}\rangle} \underline{\partial}^{\langle\underline{l}\rangle}=\left\langle\begin{array}{c}
\underline{k}+\underline{l} \\
\underline{k}
\end{array}\right\rangle \partial^{\langle\underline{k}+\underline{l}\rangle} \quad \text { with } \quad\left\langle\begin{array}{c}
\underline{k}+\underline{l} \\
\underline{k}
\end{array}\right\rangle=\frac{\left(\begin{array}{c}
\underline{k}+\underline{l} \\
\underline{k}
\end{array}\right)}{\left\{\frac{\underline{k}+\underline{l}}{\underline{k}}\right\}} .
$$

We also have

$$
\underline{\partial}^{\langle\underline{k}\rangle} f=\sum_{\underline{i} \leq \underline{k}}\left\{\begin{array}{l}
\underline{k} \\
\underline{i}
\end{array}\right\} \underline{\partial}^{\langle\underline{i}\rangle}(f) \underline{\partial}^{\langle\underline{k}-\underline{i}\rangle} .
$$

In this last formula, we implicitly make $\mathcal{D}_{X}^{(m)}$ act on $\mathcal{O}_{X}$. This is formally obtained as follows: a differential operator of order $n$ is nothing but a linear map $P: \mathcal{P}_{X, m}^{n} \rightarrow$ $\mathcal{O}_{X}$ and we compose it on the left with the map induced by the second projection $p_{2}^{*}: \mathcal{O}_{X} \rightarrow \mathcal{P}_{X, m}^{n}$.

For example, if we work locally, then $\underline{t} \underline{h}$ is sent by $p_{2}^{*}$ to

$$
(\underline{t}+\underline{\tau})^{\underline{h}}=\sum_{\underline{k}}(\underline{\underline{h}}) t^{\underline{h}-\underline{k}} \tau^{\underline{k}}=\sum_{\underline{k}} q_{\underline{k}} !\left(\frac{\underline{h}}{\underline{k}}\right) t^{\underline{h}-\underline{k}} \tau^{\{\underline{k}\}}
$$

and therefore,

$$
\underline{\partial}^{\langle\underline{k}\rangle}\left(\underline{t}^{\underline{h}}\right)=q_{\underline{k}} !\left(\frac{h}{\underline{k}}\right) t^{\underline{h}-\underline{k}} .
$$

Finally, note that $\mathcal{D}_{X}^{(0)}$ is locally generated by $\partial_{1}, \ldots, \partial_{r}$ and that $\mathcal{D}_{X}^{(\infty)}$ is Grothendieck's ring of differential operators. In general, when $k<p^{m+1}$, it is convenient to define $\partial^{[k]}=\partial^{\langle k\rangle} / q_{k}$ ! and note that $\mathcal{D}_{X}^{(m)}$ is locally generated by the $\partial_{i}^{\left[p^{l}\right]}=\partial^{\left\langle p^{l}\right\rangle}$ for $l \leq m$. In particular, we see that the diamond brackets notation should not appear very often in practice.

\section{$\S 3$. The $p^{m}$-curvature map}

We assume from now on that $m \neq \infty$.

If $X$ is a scheme of characteristic $p$, we will denote by $F: X \rightarrow X$ the $m+1$-st iterate of its Frobenius endomorphism (given by the identity on $X$ and the map $f \mapsto f^{p^{m+1}}$ on functions).

Lemma 3.1. Let $X \hookrightarrow Y$ be an immersion defined by an ideal $\mathcal{I}$. Then, the map

$$
\mathcal{I} \rightarrow \mathcal{P}_{X, m}(Y), \quad \varphi \mapsto \varphi^{\left\{p^{m+1}\right\}},
$$


composed with the projection

$$
\mathcal{P}_{X, m}(Y) \rightarrow \mathcal{P}_{X, m}(Y) / \mathcal{I} \mathcal{P}_{X, m}(Y),
$$

is an $F^{*}$-linear map that is zero on $\mathcal{I}^{2}$.

Proof. If $\varphi, \psi \in \mathcal{I}$, we have

$$
(\varphi+\psi)^{\left\{p^{m+1}\right\}}=\varphi^{\left\{p^{m+1}\right\}}+\psi^{\left\{p^{m+1}\right\}}+\sum_{\substack{i+j=p^{m+1} \\
i, j>0}}\left\langle\begin{array}{c}
p^{m+1} \\
i
\end{array}\right\rangle \varphi^{\{i\}} \psi^{\{j\}} .
$$

When $0<i, j<p^{m+1}$, we have $q_{i}, q_{j}<p$ and $q_{i}$ ! and $q_{j}$ ! are therefore invertible. It follows that the last part in the sum falls into $\mathcal{I}^{p^{m+1}}$. In particular, it is zero modulo $\mathcal{I P}_{X, m}(Y)$ and it follows that the composite map is additive.

Also, clearly, if $f \in \mathcal{O}_{X}$ and if $\varphi \in \mathcal{I}$, then $f \varphi$ is sent to

$$
(f \varphi)^{\left\{p^{m+1}\right\}}=f^{p^{m+1}} \varphi^{\left\{p^{m+1}\right\}}=F^{*}(f) \varphi^{\left\{p^{m+1}\right\}} .
$$

And we see that the map is $F^{*}$-linear. Finally, if $\varphi, \psi \in \mathcal{I}$, then $\varphi \psi$ is sent to

$$
(\varphi \psi)^{\left\{p^{m+1}\right\}}=\varphi^{p^{m+1}} \psi^{\left\{p^{m+1}\right\}} \in \mathcal{I P}_{X, m}(Y) .
$$

For the rest of this section, we fix a base scheme $S$ of characteristic $p$ and we assume that $X$ is an $S$-scheme. We consider the usual commutative diagram with cartesian square (recall that here $F$ denotes the $m+1$-st iteration of Frobenius)

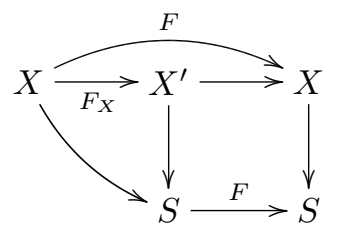

If we apply Lemma 3.1 to the case of the diagonal embedding of $X$ in $X \times_{S} X$, we obtain, after linearizing and since $F_{X}^{*} \Omega_{X^{\prime}}^{1}=F^{*} \Omega_{X}^{1}$, an $\mathcal{O}_{X}$-linear map

$$
F_{X}^{*} \Omega_{X^{\prime}}^{1} \rightarrow \mathcal{P}_{X, m} / \mathcal{I}_{X, m}
$$

which we will call divided Frobenius.

We may now prove the level $m$ version of Mochizuki's theorem ([11, Proposition 1.7]):

Proposition 3.2. If $X$ is a smooth $S$-scheme, the divided Frobenius extends uniquely to an isomorphism of $\mathcal{O}_{X}$-modules

$$
F_{X}^{*} \Omega_{X^{\prime} / S}^{1} \simeq \mathcal{I}_{X, m} \mathcal{P}_{X, m}^{p^{m+1}} / \mathcal{I} \mathcal{P}_{X, m}^{p^{m+1}}
$$


Proof. From the discussion above, it is clear that we have such a map. In order to show that it is an isomorphism, we may assume that there are local coordinates $t_{1}, \ldots, t_{r}$ on $X$, pull them back as $t_{1}^{\prime}, \ldots, t_{r}^{\prime}$ on $X^{\prime}$ and also set as usual $\tau_{i}:=$ $1 \otimes t_{i}-t_{i} \otimes 1$. Then, our map is simply

$$
\bigoplus_{i=1}^{r} \mathcal{O}_{X} \mathrm{~d} t_{i}^{\prime} \stackrel{\simeq}{\longrightarrow} \bigoplus_{i=1}^{r} \mathcal{O}_{X} \tau_{i}^{\left\{p^{m+1}\right\}}, \quad \mathrm{d} t_{i}^{\prime} \mapsto \tau_{i}^{\left\{p^{m+1}\right\}} .
$$

Actually, we can do a little better.

Proposition 3.3. If $X$ is a smooth $S$-scheme, then $\mathcal{I}_{X, m}^{\left\{p^{m+1}\right\}} \cap \mathcal{I} \mathcal{P}_{X, m}$ is stable under usual divided powers. Moreover, the divided Frobenius extends uniquely to an isomorphism of divided power $\mathcal{O}_{X}$-algebras

$$
F_{X}^{*} \Gamma \cdot \Omega_{X^{\prime} / S}^{1} \simeq \mathcal{P}_{X, m} / \mathcal{I} \mathcal{P}_{X, m} .
$$

Proof. The first question is local and we assume for the moment that it is solved. Then, by definition of the divided power algebra on a module, the above map

$$
F_{X}^{*} \Omega_{X^{\prime}}^{1} \rightarrow \mathcal{P}_{X, m} / \mathcal{I} \mathcal{P}_{X, m}
$$

extends uniquely to a morphism of divided power algebras

$$
F_{X}^{*} \Gamma_{\bullet} \Omega_{X^{\prime}}^{1}=\Gamma_{\bullet} F_{X}^{*} \Omega_{X^{\prime}}^{1} \rightarrow \mathcal{P}_{X, m} / \mathcal{I}_{X, m}
$$

Showing that it is an isomorphism is local again.

Thus, we assume that there are local coordinates $t_{1}, \ldots, t_{r}$ on $X$, we pull them back as $t_{1}^{\prime}, \ldots, t_{r}^{\prime}$ on $X^{\prime}$ and we also set as usual $\tau_{i}:=1 \otimes t_{i}-t_{i} \otimes 1$.

We have

$$
\mathcal{P}_{X, m} / \mathcal{I} \mathcal{P}_{X, m}=\mathcal{O}_{X}\left\langle\tau_{1}, \ldots, \tau_{r}\right\rangle^{(m)} /\left(\tau_{1}, \ldots, \tau_{r}\right),
$$

which is therefore a free $\mathcal{O}_{X}$-module with basis $\underline{\tau}^{\left\{\underline{k} p^{m+1}\right\}}$. The first assertion easily follows.

Moreover, our map is

$$
\mathcal{O}_{X}\left\langle\mathrm{~d} t_{1}^{\prime}, \ldots, \mathrm{d} t_{r}^{\prime}\right\rangle^{(0)} \rightarrow \mathcal{O}_{X}\left\langle\tau_{1}, \ldots, \tau_{r}\right\rangle^{(m)} /\left(\tau_{1}, \ldots, \tau_{r}\right), \quad \mathrm{d} t_{i}^{\prime} \mapsto \tau_{i}^{\left\{p^{m+1}\right\}} .
$$

And the left hand side is the free $\mathcal{O}_{X}$-module with basis $\underline{\mathrm{d} t^{\prime}}{ }^{[\underline{k}]}$. Our assertion is therefore a consequence of the first part of Lemma 3.4 below.

Lemma 3.4. In an ideal with partial divided powers of level $m$, we always have

1. For any $k \in \mathbb{N}$,

and $\frac{(k p) !}{(p !)^{k} k !} \in 1+p \mathbb{Z}$.

$$
\left(f^{\left\{p^{m+1}\right\}}\right)^{[k]}=\frac{(k p) !}{(p !)^{k} k !} f^{\left\{k p^{m+1}\right\}}
$$


2. If $t=q p^{m}+r$ with $q<p$ and $r<p^{m}$, then

$$
f^{\left\{k p^{m+1}\right\}} f^{\{t\}}=\left(\begin{array}{c}
k p+q \\
q
\end{array}\right) f^{\left\{k p^{m+1}+t\right\}}
$$

and $\left(\begin{array}{c}k p+q \\ q\end{array}\right) \in 1+p \mathbb{Z}$.

Proof. The first assertion comes from the case $m=0$ applied to $f^{p^{m}}$. And we may consider the formula

$$
\left(f^{[p]}\right)^{[k]}=\frac{(k p) !}{(p !)^{k} k !} f^{[k p]}
$$

as standard. Moreover, there exists a product formula for the factor:

$$
\frac{(k p) !}{(p !)^{k} k !}=\prod_{j=1}^{k-1}\left(\begin{array}{c}
j p+p-1 \\
p-1
\end{array}\right)
$$

and it is therefore sufficient to prove that each factor in this product falls into $1+p \mathbb{Z}$. We already know that they belong to $\mathbb{Z}$ and we have the product formula in $\mathbb{Z}_{(p)}$ :

$$
\left(\begin{array}{c}
j p+p-1 \\
p-1
\end{array}\right)=\prod_{i=1}^{p-1}\left(1+\frac{j}{i} p\right)
$$

The second assertion is even easier and comes from

$$
f^{\{u\}} f^{\{t\}}=\left\{\begin{array}{l}
u \\
t
\end{array}\right\} f^{\{u+t\}} \text { and }\left\{\begin{array}{c}
k p^{m+1}+t \\
t
\end{array}\right\}=\left(\begin{array}{c}
k p+q \\
q
\end{array}\right)=\prod_{i=1}^{q}\left(1+\frac{k}{i} p\right) .
$$

Definition 3.5. If $X$ is a smooth $S$-scheme, the $p^{m}$-curvature map is the morphism

$$
F_{X}^{*} S^{\bullet} \mathcal{T}_{X^{\prime}} \rightarrow \mathcal{D}_{X}^{(m)}
$$

obtained by duality from the composite

$$
\mathcal{P}_{X, m} \rightarrow \mathcal{P}_{X, m} / \mathcal{I} \mathcal{P}_{X, m} \simeq F_{X}^{*} \Gamma_{\bullet} \Omega_{X^{\prime}}^{1}
$$

We have to be a little careful here: first of all, we consider the induced morphisms

$$
\mathcal{P}_{X, m}^{[k]} \rightarrow F_{X}^{*} \Gamma_{\leq k} \Omega_{X^{\prime}}^{1}
$$

(with usual divided powers on $\mathcal{P}_{X, m}$ ), and then we dualize to get

$$
F_{X}^{*} S^{\leq k} \mathcal{T}_{X^{\prime}} \rightarrow \mathcal{D}_{X, k}^{(m)}
$$

and take the direct limit on both sides. 
Alternatively, we may also define the $p^{m}$-curvature to be the adjoint map

$$
S^{\bullet} \mathcal{T}_{X^{\prime}} \rightarrow F_{X *} \mathcal{D}_{X}^{(m)}
$$

We will denote by $\mathcal{Z}_{X}^{(m)}$ the center of $\mathcal{D}_{X}^{(m)}$ and by $\mathcal{Z} \mathcal{O}_{X}^{(m)}$ the centralizer of $\mathcal{O}_{X}$ in $\mathcal{D}_{X}^{(m)}$.

Proposition 3.6. If $X$ is a smooth scheme over $S$, then the $p^{m}$-curvature map induces an isomorphism of $\mathcal{O}_{X}$-algebras $F_{X}^{*} S^{\bullet} \mathcal{T}_{X^{\prime}} \simeq \mathcal{Z O}_{X}^{(m)}$ and an isomorphism of $\mathcal{O}_{X^{\prime}}$-algebras $S^{\bullet} \mathcal{T}_{X^{\prime}} \simeq F_{X *} \mathcal{Z}_{X}^{(m)}$.

Note that it will formally follow from the definition of the multiplication in $\mathcal{D}_{X}^{(m)}$ and Proposition 1.6 that the $p^{m}$-curvature map is a morphism of algebras. More precisely, this map is obtained by duality from a morphism of coalgebras. However, we need a local description in order to prove the rest of the proposition.

Proof. Both questions are local and we may therefore use local coordinates $t_{1}, \ldots, t_{r}$, pull them back to $t_{1}^{\prime}, \ldots, t_{r}^{\prime}$ on $X^{\prime}$ and denote by $\xi_{1}^{\prime}, \ldots, \xi_{r}^{\prime}$ the corresponding basis of $\mathcal{T}_{X^{\prime}}$. By construction, the $p^{m}$-curvature map is then given by

$$
\xi_{i}^{\prime k} \mapsto \partial_{i}^{\left\langle k p^{m+1}\right\rangle} .
$$

It follows from Lemma 3.4 (and duality) that

$$
\partial_{i}^{\left\langle k p^{m+1}\right\rangle}=\left(\partial_{i}^{\left\langle p^{m+1}\right\rangle}\right)^{k}
$$

and this shows that we do have a morphism of rings, which is clearly injective because we have free modules on both sides. The image is the $\mathcal{O}_{X^{\prime}}$-subalgebra generated by the $\partial_{i}^{\left\langle p^{m+1}\right\rangle}$ and this is exactly the center as Berthelot showed in Proposition 2.2.6 of [1].

The following theorem is due to Masaharu Kaneda ([7, Section 2.3], see also [4] in the case $m=0$ ) when $S$ is the spectrum of an algebraically closed field.

Theorem 3.7. Let $X$ be a smooth scheme over a scheme $S$ of positive characteristic $p$ and $F_{X}: X \rightarrow X^{\prime}$ the $m+1$-st iterate of the relative Frobenius. Let $\mathcal{D}_{X}^{(m)}$ be the ring of differential operators of level $m$ on $X / S$ and $\mathcal{Z O}_{X}^{(m)}$ the centralizer of $\mathcal{O}_{X}$. Then, there is an isomorphism of $\mathcal{Z O}_{X}^{(m)}$-algebras

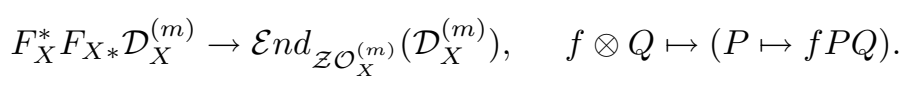

Proof. The question is local and one easily sees that $\mathcal{D}_{X / S}^{(m)}$ is free as $\mathcal{Z} \mathcal{O}_{X}^{(m)}$ module on the generators $\underline{\partial}^{\langle\underline{k}\rangle}$ with $\underline{k}<\underline{p}^{m+1}$. More precisely, this follows again 
from Lemma 3.4 that gives us, by duality,

$$
\partial_{i}^{\left\langle k p^{m+1}+t\right\rangle}=\left(\partial_{i}^{\left\langle p^{m+1}\right\rangle}\right)^{k} \partial_{i}^{\langle t\rangle}
$$

when $t<p^{m+1}$. It is then sufficient to compare bases on both sides (see Kaneda's proof for the details).

For example, when $m=0$, in the simplest case of an affine curve $X=\operatorname{Spec} A$ with coordinate $t$ and corresponding derivation $\partial$, the first powers $1, \partial, \ldots, \partial^{p-1}$ form a basis of the ring of differential operators $\mathcal{D}$ and the map of the theorem sends $\partial^{k}$ to

$$
\left[\begin{array}{cc}
0 & \partial^{p} I_{k} \\
I_{p-k} & 0
\end{array}\right] \in M_{p \times p}\left(A\left[\partial^{p}\right]\right)
$$

for $k=0, \ldots, p-1$, and $\partial^{p}$ to $\partial^{p} I_{p}$.

This theorem is usually stated as proving the Azumaya nature of $F_{X *} \mathcal{D}_{X}^{(m)}$. More precisely, we can see $F_{X *} \mathcal{D}_{X}^{(m)}$ as a sheaf of algebras on

$$
\check{\mathbf{T}}_{X^{\prime}}=\operatorname{Spec} S^{\bullet} \mathcal{T}_{X^{\prime}} \simeq \operatorname{Spec} F_{X *} \mathcal{Z}_{X}^{(m)}
$$

and the above theorem provides a trivialization of $F_{X *} \mathcal{D}_{X}^{(m)}$ along the "Frobenius"

$$
F_{X}: X \otimes_{X^{\prime}} \check{\mathbf{T}}_{X^{\prime}} \rightarrow \check{\mathbf{T}}_{X^{\prime}}
$$

Proposition 3.8. Let $X$ be a smooth $S$-scheme. If we denote by $\mathcal{K}_{X}^{(m)}$ the twosided ideal of $\mathcal{D}_{X}^{(m)}$ generated by the image of $\mathcal{T}_{X^{\prime}}$ under the $p^{m}$-curvature map, there is an exact sequence

$$
0 \rightarrow \mathcal{K}_{X}^{(m)} \rightarrow \mathcal{D}_{X}^{(m)} \rightarrow \mathcal{E} n d_{\mathcal{O}_{X^{\prime}}}\left(\mathcal{O}_{X}\right) \rightarrow 0
$$

Proof. This follows from [1, Proposition 2.2.7].

We will denote by $\widehat{\mathcal{D}}_{X}^{(m)}$ the completion of $\mathcal{D}_{X}^{(m)}$ along the two-sided ideal $\mathcal{K}_{X}^{(m)}$. We will also denote by $\widehat{\mathcal{Z}}_{X}^{(m)}$ the completion of $\mathcal{Z}_{X}^{(m)}$ along $\mathcal{Z}_{X}^{(m)} \cap \mathcal{K}_{X}^{(m)}$ and by $\widehat{\mathcal{Z}} \mathcal{O}_{X}^{(m)}$ the completion of $\mathcal{Z} \mathcal{O}_{X}^{(m)}$ along $\mathcal{Z} \mathcal{O}_{X}^{(m)} \cap \mathcal{K}_{X}^{(m)}$. Note that the $p^{m}$-curvature map gives isomorphisms

$$
F_{X}^{*} \widehat{S^{\bullet} \mathcal{T}_{X^{\prime}}} \simeq \widehat{\mathcal{Z}} \mathcal{O}_{X}^{(m)} \text { and } \widehat{S^{\bullet} \mathcal{T}_{X^{\prime}}} \simeq F_{X *} \widehat{\mathcal{Z}}_{X}^{(m)}
$$

Proposition 3.9. If $X$ is a smooth $S$-scheme, we have natural isomorphisms

$$
\widehat{\mathcal{Z}}_{X}^{(m)} \otimes_{\mathcal{Z}_{X}^{(m)}} \mathcal{D}_{X}^{(m)} \simeq \widehat{\mathcal{D}}_{X}^{(m)} \simeq \mathcal{H o m}_{\mathcal{O}_{X}}\left(\mathcal{P}_{X, m}, \mathcal{O}_{X}\right)
$$

Proof. The existence of the first map is clear and it formally follows from the definitions that it is an isomorphism. 
Now, note that the canonical projections $\mathcal{P}_{X, m} \rightarrow \mathcal{P}_{X, m}^{n}$ induce a compatible family of maps

$$
\mathcal{D}_{X, n}^{(m)}=\mathcal{H o m}_{\mathcal{O}_{X}}\left(\mathcal{P}_{X, m}^{n}, \mathcal{O}_{X}\right) \rightarrow \mathcal{H o m}_{\mathcal{O}_{X}}\left(\mathcal{P}_{X, m}, \mathcal{O}_{X}\right)
$$

from which we derive a morphism $\mathcal{D}_{X}^{(m)} \rightarrow \mathcal{H o m}_{\mathcal{O}_{X}}\left(\mathcal{P}_{X, m}, \mathcal{O}_{X}\right)$. On the other hand, using the isomorphism of Proposition 3.3 and the $p^{m}$-curvature, the projection $\mathcal{P}_{X, m} \rightarrow \mathcal{P}_{X, m} / \mathcal{I P}_{X, m}$ dualizes to

$$
\widehat{\mathcal{Z}}_{X}^{(m)} \hookrightarrow \mathcal{H o m}_{\mathcal{O}_{X}}\left(\mathcal{P}_{X, m}, \mathcal{O}_{X}\right) .
$$

And by construction, these two maps are compatible on $\mathcal{Z}_{X}^{(m)}$ and induce a map

$$
\widehat{\mathcal{Z}}_{X}^{(m)} \otimes_{\mathcal{Z}_{X}^{(m)}} \mathcal{D}_{X}^{(m)} \rightarrow \mathcal{H o m}_{\mathcal{O}_{X}}\left(\mathcal{P}_{X, m}, \mathcal{O}_{X}\right)
$$

It is now a local question to check that this is an isomorphism.

\section{$\S 4$. Lifting the $p^{m}$-curvature}

We will prove here the Azumaya nature of the ring of differential operators of higher level. In order to make it easier to read, we will not always mention direct images under Frobenius. This is not very serious because Frobenius maps are homeomorphisms and playing with direct image only affects the linearity of the maps (and this should be clear from the context).

Definition 4.1. If $X$ is a scheme of characteristic $p$, a lifting $\widetilde{X}$ of $X$ modulo $p^{2}$ is a flat $\mathbb{Z} / p^{2} \mathbb{Z}$-scheme $\tilde{X}$ such that $X=\tilde{X} \times_{\mathbb{Z} / p^{2} \mathbb{Z}} \mathbb{F}_{p}$. A lifting of a morphism $f: Y \rightarrow X$ of schemes of characteristic $p$ is a morphism $\widetilde{f}: \widetilde{X} \rightarrow \widetilde{Y}$ between liftings such that $f=\tilde{f} \times_{\mathbb{Z} / p^{2} \mathbb{Z}} \mathbb{F}_{p}$.

We will use the well known elementary result:

Lemma 4.2. If $M$ is a $\mathbb{Z} / p^{2} \mathbb{Z}$-module, multiplication by $p$ ! induces a surjective map

$$
p !: M / p M \rightarrow p M,
$$

which is bijective if $M$ is flat.

Proof. Exercise.

Note that $p !=-p \bmod p^{2}$ and this explains why minus signs will appear in the formulas below. Actually, we will need more fancy estimates: 
Lemma 4.3. We have, for any $m>0$,

$$
\left(\begin{array}{c}
p^{m+1} \\
i
\end{array}\right)=\left\{\begin{array}{ll}
1 & \text { if } i=0 \text { or } i=p^{m+1} \\
(-1)^{k} p ! & \text { if } i=k p^{m} \\
0 & \text { otherwise }
\end{array} \quad \bmod p^{2} .\right.
$$

Proof. Standard results on valuations of factorials show that

$$
v_{p}\left(\left(\begin{array}{c}
p^{m+1} \\
i
\end{array}\right)\right)= \begin{cases}0 & \text { if } i=0 \text { or } i=p^{m+1} \\
1 & \text { if } i=k p^{m} \text { with } 0<k<p \\
>1 & \text { otherwise }\end{cases}
$$

and we are therefore reduced to showing that

$$
\left(\begin{array}{c}
p^{m+1} \\
k p^{m}
\end{array}\right)=(-1)^{k} p ! \bmod p^{2},
$$

or, what is slightly easier, that

$$
\left(\begin{array}{c}
p^{m+1}-1 \\
k p^{m}-1
\end{array}\right)=(-1)^{k+1} \bmod p .
$$

First of all, we can use Lucas congruences that give

$$
\left(\begin{array}{c}
p^{m+1}-1 \\
k p^{m}-1
\end{array}\right)=\left(\begin{array}{c}
p-1 \\
k-1
\end{array}\right) \bmod p
$$

and then the binomial property

$$
\left(\begin{array}{l}
p-1 \\
k-1
\end{array}\right)+\left(\begin{array}{l}
p-1 \\
k-2
\end{array}\right)=\left(\begin{array}{c}
p \\
k-2
\end{array}\right)=0 \bmod p
$$

in order to reduce to the case $k=1$.

Up to the end of the section, we let $S$ be a scheme of characteristic $p$ and denote by $\widetilde{S}$ a lifting of $S$ (modulo $p^{2}$ as defined above).

Definition 4.4. If $X$ is an $S$-scheme, a strong lifting $\widetilde{F}: \widetilde{X} \rightarrow \widetilde{X}^{\prime}$ of the $m+1$-st iteration of Frobenius of $X$ is a morphism that satisfies

$$
f^{\prime}=1 \otimes f \bmod p \Rightarrow \widetilde{F}^{*}\left(f^{\prime}\right)=f^{p^{m+1}}+p g^{p^{m}} \text { with } g \in \mathcal{O}_{\widetilde{X}} .
$$

When $m=0$, this is nothing but a usual lifting, but the condition is stronger in general. For example, the map $t \mapsto t^{4}+2 t$ is not a strong lifting of the Frobenius on the affine line when $m=1$ and $p=2$. However, the condition is usually satisfied in practice, especially when the lifting comes from a lifting of the absolute Frobenius as the next lemma shows. 
Lemma 4.5. If $\widetilde{F}: \widetilde{X} \rightarrow \widetilde{X}$ is a lifting of the true absolute Frobenius of $X$, then for $f \in \mathcal{O}_{\tilde{X}}$, we have

$$
\widetilde{F}^{m+1 *}(f)=f^{p^{m+1}}+p g^{p^{m}}
$$

with $g \in \mathcal{O}_{\widetilde{X}}$.

Proof. By definition, we can write

$$
\widetilde{F}^{*}(f)=f^{p}+p g
$$

with $g \in \mathcal{O}_{\widetilde{X}}$ and we prove by induction on $m$ that

$$
\widetilde{F}^{m+1 *}(f)=f^{p^{m+1}}+p g^{p^{m}} .
$$

If we apply the ring homomorphism $\widetilde{F}^{*}$ on both sides of this equality, we get

$$
\begin{aligned}
\widetilde{F}^{m+2 *}(f) & =\widetilde{F}^{*}(f)^{p^{m+1}}+p \widetilde{F}^{*}(g)^{p^{m}} \\
& =\left(f^{p}+p g\right)^{p^{m+1}}+p\left(g^{p}+p h\right)^{p^{m}}=f^{p^{m+2}}+p g^{p^{m+1}} .
\end{aligned}
$$

Now, we fix a smooth $S$-scheme $X$ and let $\widetilde{F}: \widetilde{X} \rightarrow \widetilde{X}^{\prime}$ be a strong lifting of the $m+1$-st iteration of the relative Frobenius of $X$. We will denote by $\widetilde{X} \times \widetilde{X}$ (resp. $\left.\widetilde{X}^{\prime} \times \widetilde{X}^{\prime}\right)$ the fibered product over $\widetilde{S}$ and by $\widetilde{\mathcal{I}}\left(\right.$ resp. $\left.\widetilde{\mathcal{I}}^{\prime}\right)$ the ideal of $\widetilde{X}$ in $\widetilde{X} \times \widetilde{X}\left(\operatorname{resp} . \widetilde{X}^{\prime}\right.$ in $\left.\tilde{X}^{\prime} \times \widetilde{X}^{\prime}\right)$.

Lemma 4.6. Assume that $\widetilde{F}^{*}\left(f^{\prime}\right)=f^{p^{m+1}}+p g^{p^{m}}$ with $g \in \mathcal{O}_{\tilde{X}}$. Let $\varphi=1 \otimes f-$ $f \otimes 1, \varphi^{\prime}=1 \otimes f^{\prime}-f^{\prime} \otimes 1$ and $\psi=1 \otimes g-g \otimes 1$. Then, the composite map

$$
\widetilde{F}^{*}: \widetilde{\mathcal{I}}^{\prime} \hookrightarrow \mathcal{O}_{\widetilde{X}^{\prime} \times \widetilde{X}^{\prime}} \stackrel{\widetilde{F}^{*} \times \widetilde{F}^{*}}{\longrightarrow} \mathcal{O}_{\widetilde{X} \times \widetilde{X}} \rightarrow \mathcal{P}_{\widetilde{X}, m}
$$

sends $\varphi^{\prime}$ to

$$
p !\left(\varphi^{\left\{p^{m+1}\right\}}+\sum_{k=1}^{p-1}(-1)^{k} f^{(p-k) p^{m}} \varphi^{k p^{m}}-\psi^{p^{m}}\right) .
$$

Proof. We have

$$
\widetilde{F}^{*}\left(\varphi^{\prime}\right)=1 \otimes f^{p^{m+1}}-f^{p^{m+1}} \otimes 1+1 \otimes p g^{p^{m}}-p g^{p^{m}} \otimes 1
$$

(recall that $p^{2}=0$ on $\widetilde{S}$ )

$$
=\varphi^{p^{m+1}}+\sum_{i=1}^{p^{m+1}-1}\left(\begin{array}{c}
p^{m+1} \\
i
\end{array}\right) f^{p^{m+1}-i} \varphi^{i}+p \psi^{p^{m}} .
$$

We finish with Lemma 4.3 . 
Proposition 4.7. There is a well defined map

$$
\frac{1}{p !} \widetilde{F}^{*}: \widetilde{\mathcal{I}}^{\prime} \rightarrow p \mathcal{P}_{\widetilde{X}, m} \simeq \mathcal{P}_{\widetilde{X}, m} / p \mathcal{P}_{\widetilde{X}, m} \simeq \mathcal{P}_{X, m}
$$

that factors through $\Omega_{X^{\prime}}^{1}$ and takes values in $\mathcal{J}_{X, m}$. Moreover, the induced morphism

$$
\frac{1}{p !} \widetilde{F}^{*}: \Omega_{X^{\prime}}^{1} \rightarrow \mathcal{P}_{X, m}
$$

is a lifting of divided Frobenius

$$
\Omega_{X^{\prime}}^{1} \rightarrow \mathcal{P}_{X, m} / \mathcal{I} \mathcal{P}_{X, m}
$$

Proof. It follows from Lemma 4.6 that the map is well defined: more precisely, we need to check that $\widetilde{F}^{*}$ sends $\widetilde{\mathcal{I}}^{\prime}$ inside $p \mathcal{P}_{\widetilde{X}, m}$. By linearity, it is sufficient to consider the action on sections $\varphi^{\prime}$ as in the lemma.

Now, since $\widetilde{F}^{*}$ is a morphism of rings that sends $\widetilde{\mathcal{I}}^{\prime}$ to zero modulo $p$, it is clear that $\frac{1}{p !} \widetilde{F}^{*}$ will send $\widetilde{\mathcal{I}}^{\prime 2}$ to 0 . Thus, it factors through $\Omega_{\widetilde{X}^{\prime}}^{1}$. Actually, since the target is killed by $p$, it even factors through $\Omega_{X^{\prime}}^{1}$. And it falls inside $\mathcal{J}_{X, m}$ thanks to the first part.

Finally, the last assertion follows again from the explicit description of the map.

Warning: The quotient map $\mathcal{P}_{X, m} \rightarrow \mathcal{P}_{X, m} / \mathcal{I} \mathcal{P}_{X, m}$ is not compatible with the divided power structures: $\tau_{i}^{p^{m}}$ is sent to 0 but $\left(\tau_{i}^{p^{m}}\right)^{[p]}=\tau_{i}^{\left\{p^{m+1}\right\}}$ is not.

Proposition 4.8. The divided Frobenius $\frac{1}{p !} \widetilde{F}^{*}$ extends canonically to a morphism

$$
F_{X}^{*} \Gamma_{\bullet} \Omega_{X^{\prime}}^{1} \rightarrow \mathcal{P}_{X, m}
$$

By duality, we get a morphism of $\mathcal{O}_{X}$-modules

$$
\Phi_{X}: \widehat{\mathcal{D}}_{X}^{(m)} \rightarrow \widehat{\mathcal{Z}} \mathcal{O}_{X}^{(m)} \hookrightarrow \widehat{\mathcal{D}}_{X}^{(m)} .
$$

Proof. We saw in Proposition 4.7 that the morphism $\frac{1}{p !} \widetilde{F}^{*}$ takes values in $\mathcal{J}_{X, m}$ and therefore extends to a morphism of divided power algebras

$$
\Gamma \bullet \Omega_{X^{\prime}}^{1} \rightarrow \mathcal{P}_{X, m}
$$

that we can linearize. Moreover, we saw in Proposition 3.9 that

$$
\widehat{\mathcal{D}}_{X}^{(m)} \simeq \mathcal{H o m}_{\mathcal{O}_{X}}\left(\mathcal{P}_{X, m}, \mathcal{O}_{X}\right)
$$

and we also have

$$
\widehat{\mathcal{Z}} \mathcal{O}_{X}^{(m)} \simeq F_{X}^{*} \widehat{S^{\bullet} \mathcal{T}_{X^{\prime}}} \simeq F_{X}^{*} \mathcal{H o m}_{\mathcal{O}_{X^{\prime}}}\left(\Gamma \cdot \Omega_{X^{\prime}}^{1}, \mathcal{O}_{X^{\prime}}\right) \simeq \mathcal{H o m}_{\mathcal{O}_{X}}\left(F_{X}^{*} \Gamma \bullet \Omega_{X^{\prime}}^{1}, \mathcal{O}_{X}\right)
$$


Definition 4.9. The morphism $\Phi_{X}$ is called the Frobenius of $\widehat{\mathcal{D}}_{X}^{(m)}$.

We will simply write $\Phi$ when $X$ is understood from the context but we might also use $\Phi_{X}^{(m)}$ to indicate the level. Note that $\Phi$ actually depends on the choice of the strong lifting $\widetilde{F}$ of $F_{X}$.

Proposition 4.10. If we are given local coordinates $t_{1}, \ldots, t_{r}$, then

$$
\Phi\left(\underline{\partial}^{\langle\underline{u}\rangle}\right)= \begin{cases}1 & \text { if }|\underline{n}|=\underline{0}, \\ 0 & \text { if } 0<|\underline{n}|<p^{m}, \\ \frac{1}{p !} \sum_{j=1}^{r} \partial_{i}^{\left[p^{m}\right]}\left(\widetilde{F}^{*}\left(\widetilde{t_{j}^{\prime}}\right)\right) \partial_{j}^{\left\langle p^{m+1}\right\rangle} & \text { if } \underline{n}=p^{m} \mathbf{1}_{i} .\end{cases}
$$

Actually, if $\widetilde{F}^{*}\left(\widetilde{t_{j}^{\prime}}\right)={\widetilde{t_{j}}}^{p^{m+1}}+p{\widetilde{g_{j}}}^{p^{m}}$, the third expression can be rewritten

$$
-t_{i}^{(p-1) p^{m}} \partial_{i}^{\left\langle p^{m+1}\right\rangle}-\sum_{j=1}^{r} \partial_{i}\left(g_{j}\right)^{p^{m}} \partial_{j}^{\left\langle p^{m+1}\right\rangle} .
$$

Proof. The point consists in writing $\Phi\left(\underline{\partial}^{\langle\underline{n}\rangle}\right)$ in the topological $\mathcal{O}_{X}$-basis $\underline{\partial}^{\left\langle\underline{k} p^{m+1}\right\rangle}$ of $\widehat{\mathcal{Z}} \mathcal{O}_{X}^{(m)}$ when $|\underline{n}| \leq p^{m}$. By duality, the coefficient of $\underline{\partial}^{\left\langle\underline{k}^{m+1}\right\rangle}$ in $\Phi\left(\underline{\partial}^{\langle\underline{n}\rangle}\right)$ is identical to the coefficient of $\underline{\tau}^{\{\underline{n}\}}$ in the image of $1 \otimes(\underline{\mathrm{d} t})^{[\underline{k}]}$ under the morphism

$$
\mathcal{O}_{X} \otimes_{\mathcal{O}_{X^{\prime}}} \Gamma \Omega_{X^{\prime}}^{1} \rightarrow \mathcal{P}_{X, m}
$$

Recall that this is exactly

$$
\left(\frac{\widetilde{F}^{*}}{p !}\left({\widetilde{\tau^{\prime}}}^{\prime}\right)\right)^{[k]} .
$$

Since we consider only the case $|\underline{n}| \leq p^{m}$ we may work modulo $\mathcal{I}^{\left\{p^{m}+1\right\}}$ on the right. If we write $\widetilde{F}^{*}\left(\widetilde{t_{j}^{\prime}}\right)={\widetilde{t_{j}}}^{p^{m+1}}+p{\widetilde{g_{j}}}^{p^{m}}$, we obtain

$$
\begin{aligned}
\frac{1}{p !} \widetilde{F}^{*}\left(\widetilde{\tau}_{j}^{\prime}\right) & =\frac{1}{p !} \sum_{\underline{s} \neq 0} \underline{\partial}^{\langle\underline{s}\rangle}\left(\widetilde{F}^{*}\left(\widetilde{t}_{j}^{\prime}\right)\right) \tau^{\{\underline{s}\}} \\
& =-t_{j}^{(p-1) p^{m}} \tau_{j}^{p^{m}}-\sum_{i=1}^{r} \partial_{i}\left(g_{j}\right)^{p^{m}} \tau_{i}^{p^{m}} \bmod \mathcal{I}^{\left\{p^{m}+1\right\}}
\end{aligned}
$$

Thus, we see that the only contributions will come from the case $|\underline{k}| \leq 1$ and that, when $\underline{k} \neq \underline{0}$, the coefficient of $\underline{\tau}^{\{\underline{n}\}}$ is zero unless $\underline{n}=p^{m} \mathbf{1}_{i}$. Then, there are two cases: first $i \neq j$, in which case only

$$
\frac{1}{p !} \partial_{i}^{\left[p^{m}\right]}\left(\widetilde{F}^{*}\left(\widetilde{t_{j}^{\prime}}\right)\right)=-\partial_{i}\left(g_{j}\right)^{p^{m}}
$$


is left, and the case $i=j$ where we obtain

$$
\frac{1}{p !} \partial_{i}^{\left[p^{m}\right]}\left(\widetilde{F}^{*}\left(\widetilde{t}_{i}^{\prime}\right)\right)=-t_{i}^{(p-1) p^{m}}-\partial_{i}\left(g_{i}\right)^{p^{m}} .
$$

For example, when $m=0$, in the case of the affine line with parameter $t$ and derivation $\partial$, if we choose the usual lifting of Frobenius $t \mapsto t^{p}$, we obtain the simple formula $\Phi(\partial)=-t^{p-1} \partial^{p}$.

Formulas are a lot more complicated in general but they become surprisingly nice when we stick to the usual generators of the center.

Proposition 4.11. For all $i=1, \ldots, r$, we have

$$
\Phi\left(\partial_{i}^{\left\langle p^{m+1}\right\rangle}\right)=\partial_{i}^{\left\langle p^{m+1}\right\rangle}+\Phi\left(\partial_{i}^{\left\langle p^{m}\right\rangle}\right)^{p} .
$$

Proof. As above, the coefficient of $\underline{\partial}^{\left\langle\underline{k} p^{m+1}\right\rangle}$ in $\Phi\left(\partial_{i}^{\left\langle p^{m+1}\right\rangle}\right)$ is identical to the coefficient of $\tau_{i}^{\left\{p^{m+1}\right\}}$ in

$$
\frac{\widetilde{F}^{*}}{p !}\left({\tilde{\tau^{\prime}}}^{\prime[\underline{k}]}=\prod_{j=1}^{r}\left(\tau_{j}^{\left\{p^{m+1}\right\}}+\sum_{l=1}^{p-1}(-1)^{l} t_{j}^{(p-l) p^{m}} \tau_{j}^{l p^{m}}-\sum_{l=1}^{r} \partial_{l}\left(g_{j}\right)^{p^{m}} \tau_{l}^{p^{m}}\right)^{\left[k_{j}\right]}\right.
$$

if we write $\widetilde{F}^{*}\left(\widetilde{t_{j}^{\prime}}\right)={\widetilde{t_{j}}}^{p^{m+1}}+p{\widetilde{g_{j}}}^{p^{m}}$.

Thus, we see that the only contributions will come from the cases $\underline{k}=\mathbf{1}_{i}$ that gives $\tau_{i}^{\left\{p^{m+1}\right\}}$ and $\underline{k}=p \mathbf{1}_{j}$ that will give

$$
\left(-\partial_{i}\left(g_{j}\right)^{p^{m}} \tau_{i}^{p^{m}}\right)^{[p]}=\left(-\partial_{i}\left(g_{j}\right)^{p^{m}}\right)^{p} \tau_{i}^{\left\{p^{m+1}\right\}}
$$

for all $j$ plus the special contribution

$$
\left(-t_{i}^{(p-1) p^{m}} \tau_{i}^{p^{m}}\right)^{[p]}=\left(-t_{i}^{(p-1) p^{m}}\right)^{p} \tau_{i}^{\left\{p^{m+1}\right\}}
$$

of the case $j=i$. In other words, we obtain

$$
\Phi\left(\partial_{i}^{\left\langle p^{m+1}\right\rangle}\right)=\partial_{i}^{\left\langle p^{m+1}\right\rangle}+\left(-t_{i}^{(p-1) p^{m}}\right)^{p} \partial_{i}^{\left\langle p^{m+2}\right\rangle}+\sum_{j=1}^{r}\left(-\partial_{i}\left(g_{j}\right)^{p^{m}}\right)^{p} \partial_{j}^{\left\langle p^{m+2}\right\rangle} .
$$

Our assertion therefore follows from the formulas in Proposition 4.10 because, thanks to Lemma 3.4, we have

$$
\left(\partial_{j}^{\left\langle p^{m+1}\right\rangle}\right)^{p}=\partial_{j}^{\left\langle p^{m+2}\right\rangle} .
$$

This calculation shows in particular that $\Phi$ is not a morphism of rings. However, we will see later on that the map induced by $\Phi$ on the center is a morphism of rings so that the above formula fully describes this map. 
We recall now the following general result on Frobenius and divided powers:

Lemma 4.12. The canonical map $P_{X, m} \rightarrow X \times X$ factors through $X \times_{X^{\prime}} X$ if $X$ is seen as an $X^{\prime}$-scheme via $F_{X}$. Actually, if we are given local coordinates $t_{1}, \ldots, t_{r}$ and we set $\tau_{i}:=1 \otimes t_{i}-t_{i} \otimes 1$, the corresponding map on sections is the canonical injection

$$
\mathcal{O}_{X}[\underline{\tau}] /\left(\underline{\tau}^{p^{m+1}}\right) \hookrightarrow \mathcal{O}_{X}\langle\underline{\tau}\rangle^{(m)} .
$$

Proof. In order to prove the first assertion, the point is to check that if $f \in \mathcal{O}_{X}$, then $1 \otimes f^{p^{m+1}}-f^{p^{m+1}} \otimes 1$ is sent to zero in $\mathcal{P}_{X, m}$. But we have

$$
1 \otimes f^{p^{m+1}}-f^{p^{m+1}} \otimes 1=p !(1 \otimes f-f \otimes 1)^{\left\{p^{m+1}\right\}}=0 .
$$

Concerning the second assertion, we just have to verify that

$$
\mathcal{O}_{X}[\underline{\tau}] /\left(\underline{\tau}^{p^{m+1}}\right) \simeq \mathcal{O}_{X \times_{X^{\prime}} X}
$$

Since Frobenius is cartesian on étale maps ([6, XIV, 1, Proposition 2]), we may assume that $X=\mathbf{A}_{S}^{r}$, in which case this is clear.

Our main theorem comes now:

Theorem 4.13. Let $X$ be a smooth scheme over a scheme $S$ of positive characteristic $p$ and $\widetilde{F}$ a strong lifting of the $m+1$-st iterate of the relative Frobenius of $X$. The divided Frobenius extends canonically to an isomorphism

$$
\mathcal{O}_{X \times_{X^{\prime}} X} \otimes \mathcal{O}_{X^{\prime}}, \Gamma \bullet \Omega_{X^{\prime}}^{1} \simeq \mathcal{P}_{X, m}
$$

By duality, we obtain an isomorphism of $\mathcal{O}_{X}$-algebras

$$
\widehat{\mathcal{D}}_{X}^{(m)} \simeq \mathcal{E} n d_{\widehat{\mathcal{Z}}_{X}^{(m)}}\left(\widehat{\mathcal{Z}} \mathcal{O}_{X}^{(m)}\right) .
$$

Proof. Thanks to Lemma 4.12, we may extend by linearity the morphism of divided power algebras

$$
\Gamma \cdot \Omega_{X^{\prime}}^{1} \rightarrow \mathcal{P}_{X, m}
$$

and obtain

$$
\mathcal{O}_{X \times_{X^{\prime}} X} \otimes_{\mathcal{O}_{X^{\prime}}} \Gamma \bullet \Omega_{X^{\prime}}^{1} \rightarrow \mathcal{P}_{X, m}
$$

We now show that it is an isomorphism. This is a local question and we may therefore fix some coordinates $t_{1}, \ldots, t_{r}$, call $t_{1}^{\prime}, \ldots, t_{r}^{\prime}$ the corresponding coordinates on $X^{\prime}$, and as usual set $\tau_{i}:=1 \otimes t_{i}-t_{i} \otimes 1$. It follows from Proposition 4.7 that $\mathrm{d} t_{i}^{\prime}$ is sent to $\tau_{i}^{\left\{p^{m+1}\right\}}+\phi_{i}$ with

$$
\phi_{i} \in\left(\underline{\tau}^{\left(p^{m}\right)}\right) \mathcal{O}_{X}[\underline{\tau}] /\left(\underline{\tau}^{p^{m+1}}\right) .
$$


And we have to check that the divided power morphism of $\mathcal{O}_{X}[\underline{\tau}] /\left(\underline{\tau}^{p^{m+1}}\right)$-algebras

$$
\mathcal{O}_{X}[\underline{\tau}] /\left(\underline{\tau}^{p^{m+1}}\right)\left\langle\underline{\mathrm{d} t^{\prime}}\right\rangle^{(0)} \rightarrow \mathcal{O}_{X}\langle\underline{\tau}\rangle^{(m)}, \quad \mathrm{d} t_{i}^{\prime} \mapsto \tau_{i}^{\left\{p^{m+1}\right\}}+\phi_{i},
$$

is bijective. This is easy because we have free modules with explicit bases on both sides.

Now, we obtain our assertion by duality. Using the fact that $X$ is finite flat over $X^{\prime}$, so that $\mathcal{O}_{X}$ is locally free over $\mathcal{O}_{X^{\prime}}$, we have the following sequence of isomorphisms:

$$
\begin{aligned}
& \mathcal{H o m}_{\mathcal{O}_{X}}\left(\mathcal{O}_{X \times_{X^{\prime}} X} \otimes_{\mathcal{O}_{X^{\prime}}} \Gamma \cdot \Omega_{X^{\prime}}^{1}, \mathcal{O}_{X}\right) \\
& \simeq \mathcal{H o m}_{\mathcal{O}_{X^{\prime}}}\left(\Gamma \cdot \Omega_{X^{\prime}}^{1}, \mathcal{H o m}_{\mathcal{O}_{X}}\left(\mathcal{O}_{X \times_{X^{\prime}} X}, \mathcal{O}_{X}\right)\right) \\
& \simeq \mathcal{H o m}_{\mathcal{O}_{X^{\prime}}}\left(\Gamma_{\bullet} \Omega_{X^{\prime}}^{1}, \mathcal{E} n d_{\mathcal{O}_{X^{\prime}}}\left(\mathcal{O}_{X}\right)\right) \\
& \simeq \mathcal{H o m}_{\mathcal{O}_{X^{\prime}}}\left(\Gamma \cdot \Omega_{X^{\prime}}^{1}, \mathcal{O}_{X^{\prime}}\right) \otimes_{\mathcal{O}_{X^{\prime}}} \mathcal{E} n d_{\mathcal{O}_{X^{\prime}}}\left(\mathcal{O}_{X}\right) \\
& \simeq \widehat{S^{\bullet} \mathcal{T}_{X^{\prime}}} \otimes_{\mathcal{O}_{X^{\prime}}} \mathcal{E} n d_{\mathcal{O}_{X^{\prime}}}\left(\mathcal{O}_{X}\right) \simeq \mathcal{E} n d_{\widehat{S^{\bullet} \mathcal{T}_{X}^{\prime}}}\left(\mathcal{O}_{X} \otimes_{\mathcal{O}_{X^{\prime}}} \widehat{S^{\bullet} \mathcal{T}_{X^{\prime}}}\right)
\end{aligned}
$$

and we know that $S^{\bullet} \mathcal{T}_{X^{\prime}} \simeq F_{X *} \mathcal{Z}_{X}^{(m)}$.

It remains to show that this is a morphism of rings and we do that by proving that it comes by duality from a morphism of coalgebras. Actually, both morphisms

$$
\mathcal{O}_{X \times_{X^{\prime}} X} \rightarrow \mathcal{P}_{X, m} \quad \text { and } \quad \Gamma_{\bullet} \Omega_{X^{\prime}}^{1} \rightarrow \mathcal{P}_{X, m}
$$

are compatible with the coalgebra structures. We can be more precise: for the first one, this is because the comultiplication is induced on both sides by the same formula

$$
f \otimes g \mapsto f \otimes 1 \otimes g
$$

and for the second one, it is an immediate consequence of the universal property of divided powers.

Warning: The isomorphism of the theorem is not a morphism of $\widehat{\mathcal{Z}}_{X}^{(m)}$-algebras. However, we have the following:

Corollary 4.14. The morphism $\Phi$ induces an automorphism of the ring $\widehat{\mathcal{Z}}_{X}^{(m)}$ (that depends on the lifting of Frobenius).

Proof. The isomorphism of rings

$$
\widehat{\mathcal{D}}_{X}^{(m)} \simeq \mathcal{E} n d_{\widehat{\mathcal{Z}}_{X}^{(m)}}\left(\widehat{\mathcal{Z}} \mathcal{O}_{X}^{(m)}\right)
$$

induces an isomorphism on the centers which is nothing but the map induced by $\Phi$. 
Corollary 4.15. The sheaf $\widehat{\mathcal{Z}} \mathcal{O}_{X}^{(m)}$ is a (left) $\widehat{\mathcal{D}}_{X}^{(m)}$-module for the action

$$
P \bullet f Q=\Phi(P f) Q, \quad P \in \widehat{\mathcal{D}}_{X}^{(m)}, f \in \mathcal{O}_{X}, Q \in \widehat{\mathcal{Z}}_{X}^{(m)}
$$

(that again depends on the lifting of Frobenius).

Proof. Using the fact that the action is $\widehat{\mathcal{Z}} \mathcal{O}_{X}^{(m)}$-linear by definition, we may assume that $Q=1$. And since the isomorphism

$$
\widehat{\mathcal{D}}_{X}^{(m)} \simeq \mathcal{E} n d_{\widehat{\mathcal{Z}}_{X}^{(m)}}\left(\widehat{\mathcal{Z}} \mathcal{O}_{X}^{(m)}\right)
$$

is $\mathcal{O}_{X}$-linear on both sides, we may assume that $f=1$. We are therefore reduced to checking that $P \bullet 1=\Phi(P)$, which follows from the definition of the map.

For example, when $m=0$, in the case of the affine line with parameter $t$ and derivation $\partial$ and if we choose the usual lifting of Frobenius $t \mapsto t^{p}$, we have, for $k>0$,

$$
\partial \bullet t^{k}=k t^{k-1}-t^{k} t^{p-1} \partial^{p}=\left(k-t^{p} \partial^{p}\right) t^{k-1} .
$$

Thus, if we use $\left(1, t, \ldots, t^{p-1}\right)$ as a basis of $k[t]\left[\left[\partial^{p}\right]\right]$ over $k\left[t^{p}\right]\left[\left[\partial^{p}\right]\right]$, we see that $\partial$ acts as

$$
\left[\begin{array}{ccccc}
0 & -t^{p} \partial^{p}+1 & 0 & \cdots & 0 \\
\vdots & 0 & \ddots & \ddots & \vdots \\
\vdots & \vdots & \ddots & \ddots & 0 \\
0 & 0 & \cdots & 0 & -t^{p} \partial^{p}+p-1 \\
-\partial^{p} & 0 & \cdots & \cdots & 0
\end{array}\right]
$$

Warning: Scalar restriction to $\widehat{\mathcal{Z}}_{X}^{(m)}$ of the action of $\widehat{\mathcal{D}}_{X}^{(m)}$ on $\widehat{\mathcal{Z}} \mathcal{O}_{X}^{(m)}$ is different from the natural action of $\widehat{\mathcal{Z}}_{X}^{(m)}$.

The last result shows that the action of $\widehat{\mathcal{D}}_{X}^{(m)}$ on $\widehat{\mathcal{Z}} \mathcal{O}_{X}^{(m)}$ and the Frobenius of $\widehat{\mathcal{D}}_{X}^{(m)}$ completely determine each other. For computations, since the action is $\widehat{\mathcal{Z}}_{X}^{(m)}$ linear and $\widehat{\mathcal{D}}_{X}^{(m)}$ is generated by the operators of order at most $p^{m}$, the following result might be useful.

Proposition 4.16. If $P \in \widehat{\mathcal{D}}_{X}^{(m)}$ has order at most $p^{m}$, and $f \in \mathcal{O}_{X}$, then

$$
P \bullet f=P(f)+f \Phi(P) .
$$

Proof. This is a local question and we may therefore assume that we have local coordinates $t_{1}, \ldots, t_{r}$. By linearity, it is sufficient to show that for $|\underline{n}| \leq p^{m}$, we have

$$
\underline{\partial}^{\langle\underline{n}\rangle} \bullet f=\underline{\partial}^{\langle\underline{n}\rangle}(f)+f \Phi\left(\underline{\partial}^{\langle\underline{n}\rangle}\right) .
$$


We proceed as in the proof of 4.10 By definition, the image of $(1 \otimes f) \otimes(\underline{d t})^{[\underline{k}]}$ under the morphism

$$
\mathcal{O}_{X \times_{X^{\prime}} X} \otimes_{\mathcal{O}_{X^{\prime}}} \Gamma \cdot \Omega_{X^{\prime}}^{1} \rightarrow \mathcal{P}_{X, m}
$$

is exactly

$$
\epsilon(f)\left(\frac{1}{p !} \widetilde{F}^{*}\left(\tilde{\tau}^{\prime}\right)\right)^{[\underline{k}]}
$$

where $\epsilon$ denotes the Taylor series.

The coefficient of $\underline{\tau}^{\{\underline{n}\}}$ in this series is the same as the coefficient of $\underline{\partial}^{\left\{\underline{k} p^{m+1}\right\}}$ in $\underline{\partial}^{\langle\underline{n}\rangle} \bullet f$. We may work modulo $\mathcal{I}^{\left\{p^{m}+1\right\}}$ on the right and we know that, then, all coefficients in $\frac{\widetilde{F}^{*}}{p !}\left(\widetilde{\tilde{\tau}^{\prime}}\right)$ are zero unless $\underline{n}=p^{m} \mathbf{1}_{i}$. Thus, we are left with the case $\underline{k}=\underline{0}$, which gives $\underline{\partial}^{\langle\underline{h}\rangle}(f)$, and the cases $\underline{k}=\mathbf{1}_{i}$, which give the different summands of $f \Phi\left(\underline{\partial}^{\langle\underline{n}\rangle}\right)$.

Note that the formula is more complicated in higher order: for example if $X$ is the affine line over $\mathbb{F}_{2}, m=0$ and $\widetilde{F}$ is the usual lifting of Frobenius, we have

$$
\partial^{3} \bullet f=\partial(f) \Phi\left(\partial^{2}\right)+f \Phi\left(\partial^{3}\right) .
$$

\section{$\S 5$. Higgs modules}

If $u: X \rightarrow Y$ is a morphism of schemes, $\mathcal{E}$ is an $\mathcal{O}_{X}$-module and

$$
\theta: \mathcal{E} \rightarrow \mathcal{E} \otimes_{\mathcal{O}_{X}} u^{*} \Omega_{Y}^{1}
$$

is an $\mathcal{O}_{X}$-linear map, we will write

$$
\theta^{(1)}: \mathcal{E} \otimes_{\mathcal{O}_{X}} u^{*} \Omega_{Y}^{1} \stackrel{\theta \otimes \mathrm{Id}}{\longrightarrow} \mathcal{E} \otimes_{\mathcal{O}_{X}} u^{*} \Omega_{Y}^{1} \otimes_{\mathcal{O}_{X}} u^{*} \Omega_{Y}^{1} \stackrel{\operatorname{Id} \otimes \wedge}{\longrightarrow} \mathcal{E} \otimes_{\mathcal{O}_{X}} u^{*} \Omega_{Y}^{2} .
$$

Definition 5.1. Let $u: X \rightarrow Y$ be a morphism of schemes. Then, a Higgs $u$-module on $X$ is an $\mathcal{O}_{X}$-module $\mathcal{E}$ endowed with an $\mathcal{O}_{X}$-linear map

$$
\theta: \mathcal{E} \rightarrow \mathcal{E} \otimes \mathcal{O}_{X} u^{*} \Omega_{Y}^{1}
$$

such that $\theta^{(1)} \circ \theta=0$. When $u=\operatorname{Id}_{X}$, we speak of a Higgs module on $X$. When $u=F_{X}$ is a (relative iterated) Frobenius morphism, we speak of a Higgs $F$-module on $X$.

The Higgs $u$-modules form a category with compatible $\mathcal{O}_{X}$-linear maps as morphisms. Exactly as modules with integrable connections may be seen as $\mathcal{D}$ modules, we can interpret the category of Higgs $u$-modules as a category of modules over a suitable ring. 
Proposition 5.2. Let $u: X \rightarrow Y$ be a morphism of schemes with $Y$ smooth, and $\mathcal{E}$ an $\mathcal{O}_{X}$-module endowed with an $\mathcal{O}_{X}$-linear map

$$
\theta: \mathcal{E} \rightarrow \mathcal{E} \otimes_{\mathcal{O}_{X}} u^{*} \Omega_{Y}^{1}
$$

Then, $\mathcal{E}$ is a Higgs u-module if and only if the dual action

$$
u^{*} \mathcal{T}_{Y} \times \mathcal{E} \rightarrow \mathcal{E}, \quad(\xi, s) \rightarrow \xi s,
$$

extends to a structure of $u^{*} S^{\bullet} \mathcal{T}_{Y}$-module. This is an equivalence of categories.

Proof. Note first that a structure of $u^{*} S^{\bullet} \mathcal{T}_{Y}$-module is given by a homomorphism

$$
S^{\bullet} u^{*} \mathcal{T}_{Y}=u^{*} S^{\bullet} \mathcal{T}_{Y} \rightarrow \mathcal{E} n d_{\mathcal{O}_{X}}(\mathcal{E})
$$

of $\mathcal{O}_{X}$-algebras. The universal property of the symmetric algebra tells us that this is equivalent to a linear map

$$
\rho: u^{*} \mathcal{T}_{Y} \rightarrow \mathcal{E} n d_{\mathcal{O}_{X}}(\mathcal{E})
$$

satisfying $\rho(\xi) \circ \rho\left(\xi^{\prime}\right)=\rho\left(\xi^{\prime}\right) \circ \rho(\xi)$ whenever $\xi, \xi^{\prime} \in \mathcal{T}_{Y}$. Alternatively, it corresponds to a bilinear map

$$
u^{*} \mathcal{T}_{Y} \times \mathcal{E} \rightarrow \mathcal{E}, \quad(\xi, s) \mapsto \xi s,
$$

satisfying $\xi \xi^{\prime} s=\xi^{\prime} \xi s$ for $\xi, \xi^{\prime} \in \mathcal{T}_{Y}$ and $s \in \mathcal{E}$. And the corresponding map

$$
\theta: \mathcal{E} \rightarrow \mathcal{E} \otimes_{\mathcal{O}_{X}} u^{*} \Omega_{Y}^{1}
$$

is given in local coordinates by $\theta(s)=\sum \xi_{i} s \otimes \mathrm{d} x_{i}$. Now, we compute

$$
\theta^{(1)}(\theta(s))=\sum_{i<j}\left(\xi_{i} \xi_{j} s-\xi_{j} \xi_{i} s\right) \otimes \mathrm{d} x_{i} \wedge \mathrm{d} x_{j} .
$$

And we see that this is zero if and only if we always have $\xi_{i} \xi_{j} s=\xi_{j} \xi_{i} s$. This is exactly the condition we were looking for.

Note that, given a morphism $u: X \rightarrow Y$, there is an obvious pull-back morphism $u^{*}$ from Higgs modules on $Y$ to Higgs $u$-modules on $X$. There is also a restriction map from Higgs $u$-modules on $X$ to Higgs modules on $X$ that has no interest for us.

Definition 5.3. Let $X$ be a smooth scheme over a fixed scheme $S$ of characteristic $p$ and $F_{X}$ the relative $m+1$-st iterated Frobenius on $X$. If $\mathcal{E}$ is a $\mathcal{D}_{X}^{(m)}$-module, its underlying Higgs $F$-module is the one obtained by restriction along the $p^{m}$ curvature map

$$
F_{X}^{*} S^{\bullet} \mathcal{T}_{X^{\prime}} \rightarrow \mathcal{D}_{X}^{(m)}
$$


Its $p^{m}$-curvature is the corresponding $\mathcal{O}_{X}$-linear morphism

$$
\theta: \mathcal{E} \rightarrow \mathcal{E} \otimes_{\mathcal{O}_{X}} F_{X}^{*} \Omega_{X^{\prime}}^{1}
$$

Note that this definition of $p^{m}$-curvature is consistent with Definition 3.1.1 of [10. More precisely, having $p^{m}$-curvature equal to zero has the same meaning as in 10 .

Note that, locally, by definition, we have

$$
\theta(s)=\sum_{i} \partial_{i}^{\left\{p^{m+1}\right\}}(s) \otimes \mathrm{d} t_{i}^{\prime} .
$$

Definition 5.4. 1. Let $\mathcal{A}$ be a ring (in a topos) and $\mathcal{I}$ a left ideal in $\mathcal{A}$. An $\mathcal{A}$-module $\mathcal{E}$ is quasi-nilpotent if given any section $s \in \mathcal{E}$, there exists locally $N \in \mathbb{N}$ such that $\mathcal{I}^{N} s=0$.

2. Let $u: X \rightarrow Y$ be a morphism of schemes over some other scheme $S$. Then, a Higgs $u$-module $\mathcal{E}$ is quasi-nilpotent if it is so as a $u^{*} S^{\bullet} \mathcal{T}_{Y}$-module (with respect to its augmentation ideal).

3. Let $X$ is a smooth scheme over a scheme $S$ of characteristic $p$ and $\mathcal{E}$ a $\mathcal{D}_{X}^{(m)}$ module. Then $\mathcal{E}$ has quasi-nilpotent $p^{m}$-curvature if the underlying Higgs $F_{X^{-}}$ module is quasi-nilpotent.

Proposition 5.5. Let $X$ be a smooth scheme over $S$ of characteristic $p$ and $\mathcal{E}$ a $\mathcal{D}_{X}^{(m)}$-module. Then, the following are equivalent:

1. $\mathcal{E}$ is quasi-nilpotent (with respect to the ideal $\mathcal{K}_{X}^{(m)}$ ),

2. $\mathcal{E}$ is quasi-nilpotent (in the sense of [1, Section 2.3]),

3. $\mathcal{E}$ has quasi-nilpotent $p^{m}$-curvature.

Proof. All the definitions are local in nature and we may therefore assume that we have local coordinates $t_{1}, \ldots, t_{r}$ on $X$. Then the first and the third conditions both mean that, locally, we have

$$
\left(\underline{\partial}^{\left\langle p^{m+1}\right\rangle}\right)^{\underline{N}}(s)=0 \quad \text { for }|N| \gg 0 .
$$

Also, the second condition says that, locally again, we have $\partial_{i}^{\langle N\rangle}(s)=0$ for $N \gg 0$. This is equivalent to the first one because we always have, for $t<p^{m+1}$,

$$
\partial_{i}^{\left\langle k p^{m+1}+t\right\rangle}=\partial_{i}^{t}\left(\partial_{i}^{\left\langle p^{m+1}\right\rangle}\right)^{k}
$$

Note also that the categories of quasi-nilpotent modules on a ring $\mathcal{A}$ and on its completion $\widehat{\mathcal{A}}$ are identical. In particular, we can always consider the category of quasi-nilpotent $\mathcal{D}_{X}^{(m)}$-modules as a full subcategory of the category of $\widehat{\mathcal{D}}_{X}^{(m)}$ module. 
In order to go further, we will need the following standard result:

Lemma 5.6. Let $\mathcal{R}$ be a commutative ring, $\mathcal{M}$ be a locally free $\mathcal{R}$ module of finite rank, and $\mathcal{A}:=\mathcal{E}_{n d_{\mathcal{R}}}(\mathcal{M})$. Then, the functors

$$
\mathcal{E} \mapsto \mathcal{H o m}_{\mathcal{A}}(\mathcal{M}, \mathcal{E})
$$

from $\mathcal{A}$-modules to $\mathcal{R}$-modules and

$$
\mathcal{F} \mapsto \mathcal{M} \otimes_{\mathcal{R}} \mathcal{F}
$$

are quasi-inverse to each other.

Proof. This follows from the fact that the canonical maps

$$
\mathcal{M} \otimes_{\mathcal{R}} \mathcal{H o m}_{\mathcal{A}}(\mathcal{M}, \mathcal{E}) \rightarrow \mathcal{E} \quad \text { and } \quad \mathcal{F} \rightarrow \mathcal{H o m}_{\mathcal{A}}\left(\mathcal{M}, \mathcal{M} \otimes_{\mathcal{R}} \mathcal{F}\right)
$$

are both bijective.

We denote by $X$ a smooth scheme over a fixed scheme $S$ of characteristic $p$ and let $F_{X}$ be the relative $m+1$-st iterated Frobenius on $X$. We also fix a lifting $\widetilde{S}$ of $S$ modulo $p^{2}$ as well as a strong lifting $\widetilde{F}: \widetilde{X} \rightarrow \widetilde{X}^{\prime}$ of $F_{X}$ over $\widetilde{S}$. Associated to $\widetilde{F}$, we may consider the Frobenius $\Phi$ of $\widehat{\mathcal{D}}_{X}^{(m)}$ introduced in the previous section. Then, we have the following.

Proposition 5.7. There is an equivalence of categories between $\widehat{\mathcal{D}}_{X}^{(m)}$-modules and $\widehat{S^{\bullet} \mathcal{T}_{X^{\prime}}}$-modules given by

$$
\mathcal{E} \mapsto\left(F_{X *} \mathcal{E}\right)^{1-\Phi} \quad \text { and } \quad \mathcal{F} \mapsto F_{X}^{*} \mathcal{F}
$$

In order to understand this statement, it is necessary to make precise the definition of Frobenius invariants:

$$
\left(F_{X *} \mathcal{E}\right)^{1-\Phi}:=\left\{s \in \mathcal{E}: \forall P \in \widehat{\mathcal{D}}_{X}^{(m)}, \Phi(P)(s)=P(s)\right\} .
$$

Proof. Using Lemma 5.6, it follows from Theorem 4.13 that there is an equivalence of categories between $\widehat{\mathcal{D}}_{X}^{(m)}$-modules and $F_{X *} \widehat{\mathcal{Z}}_{X}^{(m)}$-modules given by

$$
\mathcal{E} \mapsto F_{X *} \mathcal{H}_{o m_{\widehat{\mathcal{D}}_{X}^{(m)}}}\left(\widehat{\mathcal{Z}} \mathcal{O}_{X}^{(m)}, \mathcal{E}\right) \quad \text { and } \quad \mathcal{F} \mapsto \widehat{\mathcal{Z}} \mathcal{O}_{X}^{(m)} \otimes_{\widehat{\mathcal{Z}}_{X}^{(m)}} \mathcal{F}
$$

We want to identify the right hand sides with the expressions in the proposition. For the second one, this is easy because

$$
F_{X}^{*} \mathcal{F} \simeq F_{X}^{*} F_{X *} \widehat{\mathcal{Z}}_{X}^{(m)} \otimes_{\widehat{\mathcal{Z}}_{X}^{(m)}} \mathcal{F} \simeq \widehat{\mathcal{Z}} \mathcal{O}_{X}^{(m)} \otimes_{\widehat{\mathcal{Z}}_{X}^{(m)}} \mathcal{F}
$$

In order to do the first one, we first check that the canonical map

$$
\mathcal{H o m}_{\widehat{\mathcal{D}}_{X}^{(m)}}\left(\widehat{\mathcal{Z}} \mathcal{O}_{X}^{(m)}, \mathcal{E}\right) \rightarrow \mathcal{E}, \quad \varphi \mapsto \varphi(1),
$$


is injective. If $\varphi(1)=0$, then $\varphi(f)=0$ for any $f \in \mathcal{O}_{X}$ by linearity. Moreover, we have the inclusion

$$
\mathcal{H o m}_{\widehat{\mathcal{D}}_{X}^{(m)}}\left(\widehat{\mathcal{Z}} \mathcal{O}_{X}^{(m)}, \mathcal{E}\right) \subset \mathcal{H o m}{\widehat{\mathcal{Z}}_{X}^{(m)}}\left(\widehat{\mathcal{Z}} \mathcal{O}_{X}^{(m)}, \mathcal{E}\right) \simeq \mathcal{H o m}_{\mathcal{O}_{X^{\prime}}}\left(\mathcal{O}_{X}, \mathcal{E}\right)
$$

and it follows that $\varphi=0$.

Now, if $s \in \mathcal{E}$, we see that the corresponding map

$$
\varphi: \widehat{\mathcal{Z}} \mathcal{O}_{X}^{(m)} \rightarrow \mathcal{E}, \quad f Q \mapsto f Q(s),
$$

is $\widehat{\mathcal{D}}_{X}^{(m)}$-linear if and only if $(\Phi(P f) Q)(s)=P((f Q)(s))$ for all $P \in \widehat{\mathcal{D}}_{X}^{(m)}, f \in \mathcal{O}_{X}$ and $Q \in \widehat{\mathcal{Z}}^{(m)}$. We may assume that $Q=1$ and $f=1$ and this shows that

$$
\left(F_{X *} \mathcal{E}\right)^{1-\Phi}=F_{X *} \mathcal{H o m}_{\widehat{\mathcal{D}}_{X}^{(m)}}\left(\widehat{\mathcal{Z}} \mathcal{O}_{X}^{(m)}, \mathcal{E}\right)
$$

Warning: The induced action of $\widehat{\mathcal{Z}}_{X}^{(m)}$ on $\widehat{\mathcal{Z}} \mathcal{O}_{X}^{(m)}$ is not the natural one: we need to compose with the automorphism induced by $\Phi$ on $\widehat{\mathcal{Z}}_{X}^{(m)}$.

We may now state the main theorem of this section (see [11, Theorem 2.8]):

Theorem 5.8. Let $X$ be a smooth scheme over $S$ of positive characteristic $p$. If there exists a strong lifting of the $m+1$-st iterate of the relative Frobenius of $X$ modulo $p^{2}$, then there is an equivalence of categories between quasi-nilpotent $\mathcal{D}_{X}^{(m)}$-modules and quasi-nilpotent Higgs modules on $X^{\prime}$ given by

$$
\mathcal{E} \mapsto\left(F_{X *} \mathcal{E}\right)^{1-\Phi} \quad \text { and } \quad \mathcal{F} \mapsto F_{X}^{*} \mathcal{F}
$$

Proof. The functor $F_{X *}$ induces an equivalence of categories between $\mathcal{Z}_{X}^{(m)}$-modules and Higgs modules on $X^{\prime}$. The theorem therefore follows from Proposition 5.7 since quasi-nilpotency is clearly preserved under the equivalence.

Warning: Under this equivalence, the $p^{m}$-curvature of $\mathcal{E}$ is not obtained as the simple pull-back of the Higgs structure of $\mathcal{F}$ along Frobenius: we also have to compose with the automorphism induced by $\Phi$ on the center.

Finally, we can give local formulas:

Proposition 5.9. If we are given local coordinates $t_{1}, \ldots, t_{r}$, and $\mathcal{F}$ is a Higgs module on $X^{\prime}$ with

$$
\theta(s)=\sum_{i=1}^{r} \xi_{i}^{\prime} s \otimes \mathrm{d} t_{i}
$$


then the $\mathcal{D}_{X}^{(m)}$-structure of $F_{X}^{*} \mathcal{F}$ is given by

$$
\partial_{i}^{\left[p^{l}\right]}(1 \otimes s)= \begin{cases}0 & \text { if } l<m, \\ \frac{1}{p !} \sum_{j=1}^{r} \partial_{i}^{\left[p^{m}\right]}\left(\widetilde{F}^{*}\left(\widetilde{t_{j}^{\prime}}\right)\right) \otimes \xi_{j}^{\prime} s & \text { if } l=m .\end{cases}
$$

Proof. Follows from Proposition 4.10 .

Unfortunately, it is much more complicated to recover $\mathcal{F}$ from $\mathcal{E}$.

\section{§6. Informal complements}

\section{$\S 6.1$. Linearizing with respect to the center}

As usual, $X$ denotes a smooth scheme over some scheme $S$ of characteristic $p$ and $F_{X}$ is the relative $m+1$-st iterated Frobenius on $X$. We also fix a lifting $\widetilde{S}$ of $S$ modulo $p^{2}$ as well as a strong lifting $\widetilde{F}: \widetilde{X} \rightarrow \widetilde{X}^{\prime}$ of $F_{X}$ over $\widetilde{S}$.

First of all, it is important to notice that, in the following commutative diagram, where all maps are $\mathcal{O}_{X}$-linear,

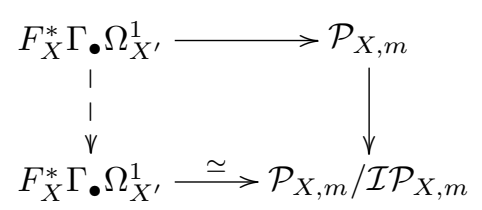

the dotted arrow is not the identity although both horizontal arrows are defined by the same map $\frac{1}{p !} \widetilde{F}$ on $\Omega_{X^{\prime}}^{1}$ and are compatible with divided powers. This is because the left arrow is not compatible with divided powers. However, one can show that the dashed arrow is bijective (it is dual to the ring automorphism induced by $\Phi$ on $\widehat{\mathcal{Z}} \mathcal{O}_{X}^{(m)}$ - see below).

Recall now that our Frobenius map

$$
\Phi: \widehat{\mathcal{D}}_{X}^{(m)} \rightarrow \widehat{\mathcal{Z}} \mathcal{O}_{X}^{(m)} \hookrightarrow \widehat{\mathcal{D}}_{X}^{(m)}
$$

induces the identity on $\mathcal{O}_{X}$ and a (non-trivial) ring automorphism of $\widehat{\mathcal{Z}}_{X}^{(m)}$. In particular, $\Phi$ restricts to an automorphism of $\widehat{\mathcal{Z}} \mathcal{O}_{X}$ and we will abusively denote by $\Phi^{-1}$ its inverse. We may then compose $\Phi^{-1}$ on the right with $\Phi$ in order to obtain a modified version of Frobenius

$$
\widetilde{\Phi}: \widehat{\mathcal{D}}_{X}^{(m)} \rightarrow \widehat{\mathcal{Z}} \mathcal{O}_{X}^{(m)} \stackrel{\Phi^{-1}}{\simeq} \widehat{\mathcal{Z}} \mathcal{O}_{X}^{(m)} \hookrightarrow \widehat{\mathcal{D}}_{X}^{(m)}
$$

that will induce the identity on $\widehat{\mathcal{Z}} \mathcal{O}_{X}^{(m)}$. 
Using this modified Frobenius gives a twisted version of our fundamental algebra isomorphism

$$
\widehat{\mathcal{D}}_{X}^{(m)} \simeq \mathcal{E} n d_{\widehat{\mathcal{Z}}_{X}^{(m)}}\left(\widehat{\mathcal{Z}} \mathcal{O}_{X}^{(m)}\right)
$$

which is now the identity on the center $\widehat{\mathcal{Z}}_{X}^{(m)}$. The corresponding $\widehat{\mathcal{D}}_{X}^{(m)}$-module structure is given by a similar formula

$$
P \widetilde{\bullet} f Q=\widetilde{\Phi}(P f) Q
$$

This twisted action is given by more complicated formulas but has the advantage of being a true Azumaya splitting for $\widehat{\mathcal{D}}_{X}^{(m)}$. Of course, one can use this twisted action in order to obtain an equivalence between quasi-nilpotent $\mathcal{D}_{X}^{(m)}$-modules and Higgs modules on $X$. Actually, $\Phi$ induces an autoequivalence of the category of quasi-nilpotent Higgs modules on $X^{\prime}$ and the twisted equivalence is obtained by composition with the old one.

\section{§6.2. Van der Put's construction}

Marius van der Put only deals with differential operators of level zero and we will therefore stick here to this case and drop $m$ from the notations. He actually works in the theory of differential fields ([13, [14]) but the translation into our language is straightforward. Also, we will not follow his notations but only try to explain his clever approach. We let $X$ be a curve over a perfect field, with a fixed coordinate $t$, and denote as usual the corresponding derivation by $\partial$.

It is important to note that van der Put works with the twisted version of the theory (see Section 6.1). The first point is to remark that the Azumaya nature of $\widehat{\mathcal{D}}_{X}$ is completely described by the element

$$
H:=\widetilde{\Phi}(\partial) \in \widehat{\mathcal{Z}} \mathcal{O}_{X}
$$

In what follows, we make $\widehat{\mathcal{D}}_{X}$ act on $\widehat{\mathcal{Z}} \mathcal{O}_{X}$ by extending trivially the action on $\mathcal{O}_{X}$ (via the isomorphism $\mathcal{O}_{X} \otimes_{\mathcal{O}_{X}}, \widehat{\mathcal{Z}}_{X} \simeq \widehat{\mathcal{Z}} \mathcal{O}_{X}$ ). Now, since the twisted action is the identity on $\widehat{\mathcal{Z}}_{X}$, we must have $\partial^{p} \widetilde{\bullet} 1=\partial^{p}$ and this leads to the condition

$$
\partial^{p-1}(H)+H^{p}=\partial^{p}
$$

Thus, van der Put's question reduces to finding such an element. He does it by successive approximations, lifting an action on the quotient $\mathcal{O}_{X}$ (see [13, Lemma 1.6]). The point is to notice that such an action corresponds to an element $h \in \mathcal{O}_{X}$ 
such that $\partial^{p-1}(h)+h^{p}=0$. In other words, he lifts the quotient isomorphism:

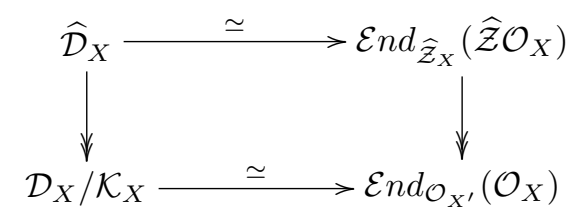

We will now check that he obtains exactly the map induced by $\widetilde{\Phi}$ if we can use $t \mapsto t^{p}$ as lifting of Frobenius modulo $p^{2}$. First of all, we have

$$
\Phi(\partial)=-t^{p-1} \partial^{p} \quad \text { and } \quad \Phi\left(\partial^{p}\right)=\partial^{p}-t^{p(p-1)} \partial^{p^{2}} .
$$

Since $\Phi$ induces a morphism of rings on $\widehat{\mathcal{Z}} \mathcal{O}_{X}$, we also have, for all $k \geq 0$,

$$
\Phi\left(\partial^{p^{k}}\right)=\partial^{p^{k}}-\left(t^{p-1}\right)^{p^{k}} \partial^{p^{k+1}}
$$

from which we derive

$$
\Phi^{-1}\left(\partial^{p}\right)=\sum_{k=1}^{\infty}\left(t^{p-1}\right)^{\frac{p^{k}-1}{p-1}-1} \partial^{p^{k}}=\sum_{k=1}^{\infty} t^{p\left(p^{k-1}-1\right)} \partial^{p^{k}} .
$$

Since $\Phi$ is $\mathcal{O}_{X}$-linear, we can deduce

$$
H:=\widetilde{\Phi}(\partial)=-t^{p-1} \Phi^{-1}\left(\partial^{p}\right)=-\sum_{k=1}^{\infty} t^{\left(p^{k}-1\right)} \partial^{p^{k}} .
$$

This is exactly the same (with different notations) as Example 1.6.1 of [13]. The reader can also look at the proof of Theorem 13.5 of [14]). Note also that many examples are worked out in that book.

\section{§6.3. Working before completion}

We keep the same notations as in Section 6.1. It is not difficult to see that the map $\Phi$ exists before completion. One just has to be careful when dualizing. This is possible because we have explicit local formulas. Thus, there is a well defined map

$$
\Phi: \mathcal{D}_{X}^{(m)} \rightarrow \mathcal{Z} \mathcal{O}_{X}^{(m)} \hookrightarrow \mathcal{D}_{X}^{(m)}
$$

Using our local formulas again, it is not difficult either to see that $\Phi$ sends $\mathcal{Z}_{X}$ into itself. But the induced map need not be bijective (in particular, we cannot define $\widetilde{\Phi}$ before completion).

For the same reasons, there exists a morphism of $\mathcal{O}_{X}$-algebras at finite level

$$
\mathcal{D}_{X}^{(m)} \rightarrow \mathcal{E}_{n d_{\mathcal{Z}_{X}^{(m)}}^{(m)}}\left(\mathcal{Z} \mathcal{O}_{X}^{(m)}\right)
$$


But again, this is not an isomorphism. However, this map is semi-linear with respect to the endomorphism $\Phi$ of $\mathcal{Z}_{X}$ and linearization provides an isomorphism

$$
\mathcal{Z}_{X} \otimes_{\Phi} \otimes_{\mathcal{Z}_{X}} \mathcal{D}_{X}^{(m)} \simeq \mathcal{E} n d_{\mathcal{Z}_{X}^{(m)}}\left(\mathcal{Z} \mathcal{O}_{X}^{(m)}\right)
$$

Note that the fact that this is an isomorphism follows from our previous results because it is a morphism of locally free $\mathcal{Z}_{X}$-modules, and that it is therefore sufficient to prove bijectivity after completing.

The endomorphism induced by $\Phi$ on $\mathcal{Z}_{X} \simeq S^{\bullet} \mathcal{T}_{X^{\prime}}$ corresponds to a morphism

$$
\alpha: \check{\mathbf{T}}_{X^{\prime}} \rightarrow \check{\mathbf{T}}_{X^{\prime}}
$$

We did not check it in general, but it is likely that $\alpha$ is surjective étale and provides an étale Azumaya splitting of $\mathcal{D}_{X}^{(m)}$ (see Proposition 2.5(1) of [11] for the case $m=0)$.

Finally, we can pullback Higgs modules along Frobenius to get a $\mathcal{D}_{X}^{(m)}$-module, but it becomes an equivalence only when restricting to quasi-nilpotent objects.

\section{§6.4. Glueing}

As before, $X$ denotes a smooth scheme over some scheme $S$ of characteristic $p$ and $F_{X}$ is the relative $m+1$-st iterated Frobenius on $X$. We also fix a lifting $\widetilde{S}$ of $S$ modulo $p^{2}$ as well as a lifting $\widetilde{X} / \widetilde{S}$ of $X / S$.

Assume for the moment that we are given two strong liftings $\widetilde{F}_{1}, \widetilde{F}_{2}: \widetilde{X} \rightarrow \widetilde{X}^{\prime}$ of $F_{X}$ over $\widetilde{S}$ (we may always twist a lifting of $X$ and therefore assume that $\widetilde{F}_{1}$ and $\widetilde{F}_{2}$ have the same domain). Considering local descriptions again, one sees that the map

$$
\widetilde{F}_{2}-\widetilde{F}_{1}: \mathcal{O}_{\widetilde{X}^{\prime}} \rightarrow \mathcal{O}_{\widetilde{X}}
$$

induces a derivation

$$
\frac{1}{p !}\left(\widetilde{F}_{2}-\widetilde{F}_{1}\right): \mathcal{O}_{X^{\prime}} \rightarrow \mathcal{O}_{X}
$$

and we obtain a natural $\mathcal{O}_{X^{\prime}}$-linear map

$$
u_{12}=\frac{1}{p !}\left(\widetilde{F}_{2}-\widetilde{F}_{1}\right): \Omega_{X^{\prime}}^{1} \rightarrow \mathcal{O}_{X}
$$

Moreover, if $\widetilde{F}_{3}$ is another strong lifting of $F_{X}$, we have (with the obvious analogous notation) $u_{13}=u_{12}+u_{23}$.

The map $u_{12}$ extends to a morphism of $\mathcal{O}_{X^{\prime}}$-algebras $S^{\bullet} \Omega_{X^{\prime}}^{1} \rightarrow \mathcal{O}_{X}$ and by linearity to a map $F_{X}^{*} S^{\bullet} \Omega_{X^{\prime}}^{1} \rightarrow \mathcal{O}_{X}$ and we finally embed into $F_{X}^{*} S^{\bullet} \Omega_{X^{\prime}}^{1}$ in order to get an endomorphism of the $\mathcal{O}_{X}$-algebra $F_{X}^{*} S^{\bullet} \Omega_{X^{\prime}}^{1}$ that we still call $u_{12}$. We may then define $\phi_{12}=\mathrm{Id}-u_{12}$ and remark that $\phi_{12} \phi_{23}=\phi_{13}$. It follows that, 
when there are only local liftings of Frobenius, we obtain glueing data and we will denote by $\mathcal{A}_{X}^{(m)}$ the resulting $\mathcal{O}_{X}$-module which is locally isomorphic to $F_{X}^{*} S^{\bullet} \Omega_{X^{\prime}}^{1}$.

In order to dualize, we introduce the completed divided power envelopes

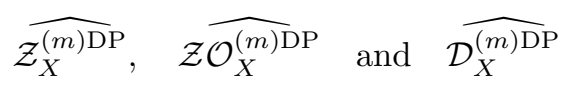

of

$$
\mathcal{Z}_{X}^{(m)}, \quad \mathcal{Z} \mathcal{O}_{X}^{(m)} \text { and } \mathcal{D}_{X}^{(m)}
$$

respectively along the augmentation ideal of $\mathcal{Z}_{X}^{(m)}$. Note that we have

$$
\widehat{\mathcal{Z}_{X}^{(m) \mathrm{DP}}} \simeq \widehat{\Gamma} \cdot \mathcal{T}_{X^{\prime}}, \quad \widehat{\mathcal{Z} \mathcal{O}_{X}^{(m) \mathrm{DP}}} \simeq \widehat{\mathcal{Z}_{X}^{(m) \mathrm{DP}}} \otimes_{\mathcal{Z}_{X}^{(m)}} \mathcal{Z} \mathcal{O}_{X}^{(m)}
$$

and

$$
\widehat{\mathcal{D}_{X}^{(m) \mathrm{DP}}} \simeq \widehat{\mathcal{Z}_{X}^{(m) \mathrm{DP}}} \otimes_{\mathcal{Z}_{X}^{(m)}} \mathcal{D}_{X}^{(m)}
$$

Thus, dual to $\mathcal{A}_{X}^{(m)}$ we obtain an $\mathcal{O}_{X}$-module $\mathcal{B}_{X}^{(m)}$, which is in fact a locally free

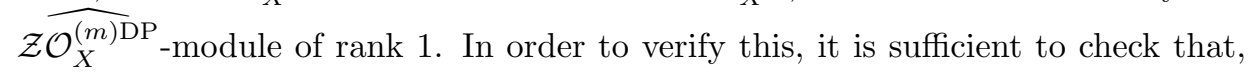
locally, the dual glueing map

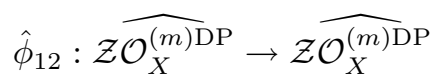

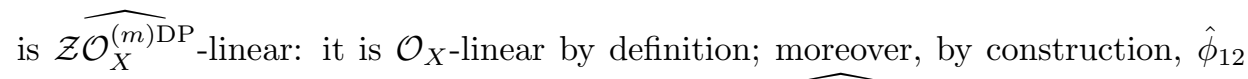
acts as the identity on the augmentation ideal of $\widehat{\mathcal{Z}_{X}^{(m) \mathrm{DP}}}$ because $u_{12}$ sends the augmentation ideal of $S^{\bullet} \Omega_{X^{\prime}}^{1}$ into $\mathcal{O}_{X}$.

Note that, locally, $\widetilde{F}_{2}-\widetilde{F}_{1}$ sends $\widetilde{\mathcal{I}}$ into $p \mathcal{O}_{\widetilde{X} \times_{\widetilde{X}^{\prime}} \widetilde{X}}$ and that we obtain a map

$$
\Omega_{X^{\prime}}^{1} \stackrel{U_{12}}{\longrightarrow} \mathcal{O}_{X \times X^{\prime} X}, \quad \omega^{\prime} \mapsto u_{12}\left(\omega^{\prime}\right) \otimes 1-1 \otimes u_{12}\left(\omega^{\prime}\right) .
$$

A construction analogous to the above provides an automorphism Id $-U_{12}$ of $\mathcal{O}_{X \times_{X^{\prime}} X} \otimes S^{\bullet} \Omega_{X^{\prime}}^{1}$ giving rise to a commutative diagram

$$
\mathcal{O}_{X \times_{X^{\prime}} X} \otimes S^{\bullet} \Omega_{X^{\prime}}^{1} \longrightarrow \mathcal{O}_{X_{X_{X^{\prime}} X}} \otimes S^{\bullet} \Omega_{\mathcal{P}_{X, m}}^{1}
$$

Dualizing provides a morphism

$$
\widehat{\mathcal{D}}_{X}^{(m)} \rightarrow \mathcal{E} n d_{\mathcal{Z}_{X}^{(m) \mathrm{DP}}}\left(\mathcal{B}_{X}^{(m)}\right),
$$


and extending scalars, a map which is an isomorphism

$$
\widehat{\mathcal{D}_{X}^{(m) \mathrm{DP}}} \simeq \mathcal{E} n d_{\widehat{\mathcal{Z}_{X}^{(m) \mathrm{DP}}}}\left(\mathcal{B}_{X}^{(m)}\right)
$$

as one easily checks.

\section{§6.5. Ogus-Vologodsky's construction}

We keep the same notations as before but we assume that $m=0$ and we drop it from the notations. Also, in what follows, there will be some sign differences with the article of Ogus and Vologodsky ([1]), due to the fact that they choose to divide by $p$ instead of $p$ ! and that $p !=-p \bmod p^{2}$.

They start with a global definition of $\mathcal{A}_{X}$ that we do not want to recall here ([11, Theorem 1.1]). In order to obtain a local description of this object, they introduce the notion of "splitting of Cartier" $\zeta: \Omega_{X^{\prime}}^{1} \rightarrow \Omega_{X}^{1}$. When $\widetilde{F}$ is a lifting of Frobenius, the standard example of such a splitting is given by Mazur's construction and is simply the composite of the map

$$
\frac{\widetilde{F}}{p !}: \Omega_{X^{\prime}}^{1} \rightarrow \mathcal{I}_{X}
$$

with the canonical projection $\mathcal{I}_{X} \rightarrow \mathcal{I}_{X} / \mathcal{I}_{X}^{[2]}=\Omega_{X}^{1}$. They linearize and dualize in order to obtain a morphism $\phi: \mathcal{T}_{X} \rightarrow F_{X}^{*} \mathcal{T}_{X^{\prime}}$. Thus, by construction, there is a commutative diagram

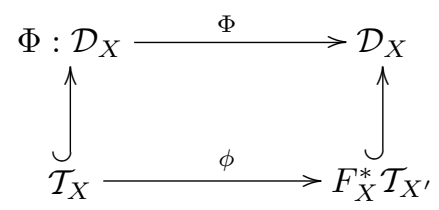

where the map on the right is the $p$-curvature map. From $\phi$, they construct a morphism $h: \check{\mathbf{T}}_{X^{\prime}} \rightarrow \check{\mathbf{T}}_{X^{\prime}}$ that is given on local sections by $h^{*}\left(\partial_{i}^{p}\right)=\Phi\left(\partial_{i}\right)^{p}$ if we use as usual the $p$-curvature map to embed $\mathcal{T}_{X^{\prime}}$ into $\mathcal{D}_{X}$. When $m=0$, our formula in Proposition 4.11 reads $\Phi\left(\partial_{i}^{p}\right)=\partial_{i}^{p}+\Phi\left(\partial_{i}\right)^{p}$ and it follows that our map $\alpha$ is the same as the one in Section 2.2 of [11. In particular, our theory is fully compatible with theirs even if the approach might sound different.

For example, their formula (2.11.2) reads locally

$$
\nabla(1 \otimes s)=\sum_{i=0}^{r} \xi_{i}^{\prime} s \otimes \zeta\left(\mathrm{d} t_{i}^{\prime}\right)
$$


and we have, with Cartier-Mazur's splitting corresponding to a lifting $\widetilde{t}_{i}^{\prime} \mapsto \widetilde{t}_{i}^{p}+p \widetilde{g}_{i}$,

$$
\zeta\left(\mathrm{d} t_{i}^{\prime}\right)=-t_{i}^{(p-1)} \mathrm{d} t_{i}-\sum_{j=1}^{r} \partial_{j}\left(g_{i}\right) \mathrm{d} t_{j} .
$$

It follows that

$$
\partial_{i}(1 \otimes s)=-t_{i}^{(p-1)} \otimes \xi_{i}^{\prime} s-\sum_{j=1}^{r} \partial_{i}\left(g_{j}\right) \otimes \xi_{j}^{\prime} s,
$$

which is exactly what we found in Proposition 5.9 .

\section{§6.6. Frobenius descent}

When $s \in \mathbb{N}$, any divided power structure of level $m$ on an ideal $\mathcal{I}$ is a fortiori a divided power structure of higher level $m+s$. It follows that if $X \hookrightarrow Y$ is any embedding, there exists a natural map $P_{X, m}(Y) \rightarrow P_{X, m+s}(Y)$. And from this, one derives, for a smooth scheme $X$ over our fixed scheme $S$, a morphism of rings $\mathcal{D}_{X}^{(m)} \rightarrow \mathcal{D}_{X}^{(m+s)}$. It is given on local generators by $\partial_{i}^{\left[p^{l}\right]} \mapsto \partial_{i}^{\left[p^{l}\right]}$ for $l \leq m$ but this is not an injective map in general. For example, if $S$ has positive characteristic $p$ and $s>0$, then $\partial_{i}^{\left\{p^{m+1}\right\}}$ is sent to zero.

Let us be more specific in this positive characteristic situation. Given any $s$, we will denote by $X^{(s)}$ the pull-back of $X$ along the $s$-th iteration of Frobenius. For $s>0$, the kernel of the map $\mathcal{D}_{X}^{(m)} \rightarrow \mathcal{D}_{X}^{(m+s)}$ is nothing but the ideal $\mathcal{K}_{X}^{(m)}$ generated by the image of $\mathcal{T}_{X^{(m+1)}}$ under the $p^{m}$-curvature map. Moreover, it induces an isomorphism of rings

$$
\mathcal{E} n d_{\mathcal{O}_{X}(m+1)}\left(\mathcal{O}_{X}\right) \simeq \mathcal{D}_{X, p^{m+1}-1}^{(m+s)}
$$

where the right hand side is the subring of differential operators of order less than $p^{m+1}$.

We now come to the basic fact on Frobenius descent: the $s$-th iteration of Frobenius induces a morphism

$$
F^{s *}: P_{X, m+s} \rightarrow P_{X(s)}, m
$$

It is given locally by $\tau_{i}^{\{k\}_{m}} \mapsto \tau_{i}^{\left\{k p^{s}\right\}_{m+s}}$ and we obtain by duality a morphism

$$
\mathcal{D}_{X}^{(m+s)} \rightarrow F^{s *} \mathcal{D}_{X^{(s)}}^{(m)}
$$

given locally by $\partial_{i}^{\langle k\rangle} \mapsto 1 \otimes \partial_{i}^{\left\langle k / p^{s}\right\rangle}$ if $p^{s} \mid k$ and 0 otherwise. Note that $F^{s *} \mathcal{D}_{X^{(s)}}^{(m)}$ has no natural ring structure so that this morphism cannot be seen as a morphism of rings. However, it induces an isomorphism of rings $\mathcal{Z} \mathcal{O}_{X}^{(m+s))} \simeq F^{s *} \mathcal{Z O}_{X^{(s)}}^{(m)}$ compatible with the $p$-curvature maps of level $m+s$ and $m$ respectively. 
If we choose compatible strong liftings, there is an obvious commutative diagram

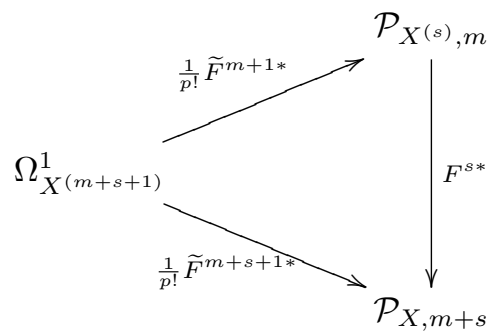

that gives by duality a commutative diagram (we ignore completions)

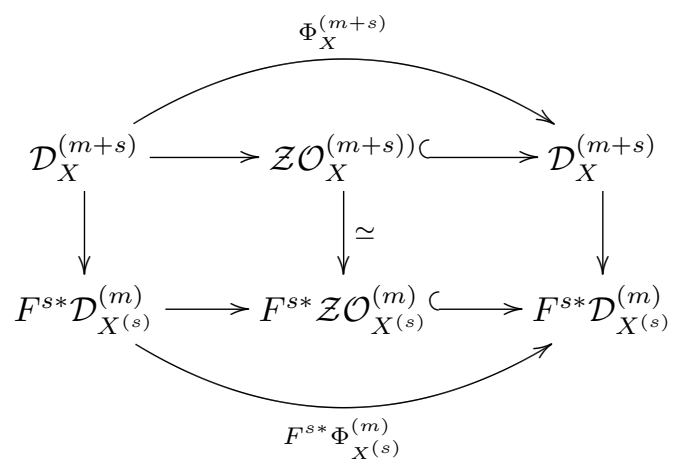

For the same reason, we have a commutative diagram

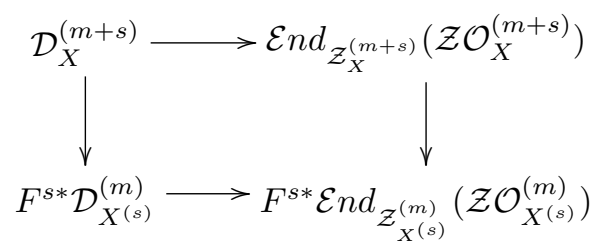

Finally, when restricting to quasi-nilpotent objects everywhere, we have compatible equivalences of categories induced by Frobenius pull-backs

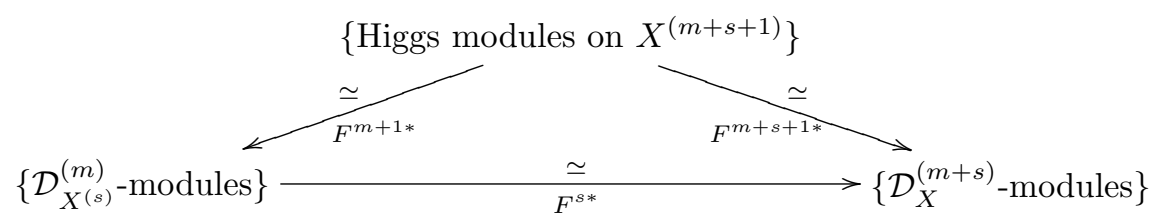

In particular, we recover our equivalence of categories as the composition of Frobenius descent and the usual case $m=0$ (again, we restrict to quasi-nilpotent ob- 
jects):

$\left\{\right.$ Higgs modules on $\left.X^{(m+1)}\right\} \underset{F^{*}}{\simeq}\left\{\mathcal{D}_{X^{(m)}}^{(0)}\right.$-modules $\} \underset{F^{m *}}{\simeq}\left\{\mathcal{D}_{X}^{(m)}\right.$-modules $\}$.

Actually, as the referee pointed out, this last equivalence (which is Theorem 2.3.6 of [2]) may be used to formally recover some of our results from [11, that is, from the case $m=0$. For example, we can obtain the description of the center as well as the Azumaya nature of $\mathcal{D}_{X}^{(m)}$ or even a construction of an étale splitting. This is because the theory of Azumaya algebras behaves remarkably well under Morita equivalence.

Let us be more precise: two rings are said to be Morita equivalent if the category of right (or equivalently left) modules on these rings are equivalent (see for example Section 18 of [9]). It then immediately follows that their centers are isomorphic since they can be identified with the rings of endomorphisms of the identity functor. And it is also easy to see that if one of them is split Azumaya, so is the other.

But there is more: being an Azumaya algebra is also invariant under Morita equivalence. We can explain this a little bit: a ring $A$ with center $Z$ is an Azumaya algebra if and only if the scalar extension functor $M \mapsto A \otimes_{Z} M$ is an equivalence between $Z$-modules and $A$-bimodules (see Theorem 5.1 .1 of [8] for example). And it is not difficult to check that if two rings are Morita equivalent, the categories of bimodules on them are equivalent.

\section{Acknowledgments}

Many thanks to Pierre Berthelot who explained to us the level zero case and helped us understand some tricky constructions. We are also grateful to the referee who gave us a positive answer to a previous query.

Adolfo Quirós was partially supported by Project GALAR (MTM2006-10548) from MEC (Spain) and by the joint Madrid Region-UAM project TENU3 (CCG08$\mathrm{UAM} / \mathrm{ESP}-3906)$.

\section{References}

[1] P. Berthelot, D-modules arithmétiques. I. Opérateurs différentiels de niveau fini, Ann. Sci. École Norm. Sup. (4) 29 (1996), 185-272. Zbl 0886.14004 MR 1373933

[2], $\mathcal{D}$-modules arithmétiques. II. Descente par Frobenius, Mém. Soc. Math. France (N.S.) 81 (2000). Zbl 0948.14017 MR 1775613

[3] P. Berthelot and A. Ogus, Notes on crystalline cohomology, Princeton Univ. Press, Princeton, NJ, 1978. Zbl 0383.14010 MR 0491705 
[4] R. Bezrukavnikov, I. Mirković, and D. Rumynin, Localization of modules for a semisimple Lie algebra in prime characteristic, Ann. of Math. (2) 167 (2008), 945-991. With an appendix by R. Bezrukavnikov and Simon Riche. Zbl pre05578707 MR 2415389

[5] G. Faltings, A p-adic Simpson correspondence, Adv. Math. 198 (2005), 847-862. Zbl 1102.14022 MR 2183394

[6] A. Grothendieck, Cohomologie l-adique et fonctions L, Lecture Notes in Math. 589, Springer, 1977. Zbl 0345.00011

[7] M. Kaneda, Direct images of $\mathcal{D}$-modules in prime characteristic, RIMS Kōkyūroku 1382 (2004), 154-170.

[8] M.-A. Knus, Quadratic and Hermitian forms over rings, Grundlehren Math. Wiss. 294, Springer, Berlin, 1991. Zbl 0756.11008 MR 1096299

[9] T. Y. Lam, Lectures on modules and rings, Grad. Texts in Math. 189, Springer, New York, 1999. Zbl 0911.16001 MR 1653294

[10] B. Le Stum and A. Quirós, Transversal crystals of finite level, Ann. Inst. Fourier (Grenoble) 47 (1997), 69-100. Zbl 0883.14006 MR 1437179

[11] A. Ogus and V. Vologodsky, Nonabelian Hodge theory in characteristic $p$, Publ. Math. Inst. Hautes Études Sci. 106 (2007), 1-138. Zbl 1140.14007 MR 2373230

[12] C. T. Simpson. Higgs bundles and local systems. Publ. Math. Inst. Hautes Études Sci. 75 (1992), 5-95. Zbl 0814.32003 MR 1179076

[13] M. van der Put. Differential equations in characteristic p, Compos. Math. 97 (1995), 227251. Zbl 0861.12004 MR 1355126

[14] M. van der Put and M. F. Singer, Galois theory of linear differential equations, Grundlehren Math. Wiss. 328, Springer, Berlin, 2003. Zbl 1036.12008 MR 1960772 